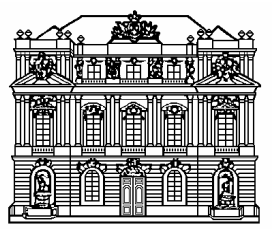

des theoretisch wissenschaftlichen Teiles

\title{
10 Anhang
}

\section{Projektwerber und Projektleitung:}

INSTITUT FÜR STRASSEN- UND VERKEHRSWESEN (ISV)

Technische Universität Graz

Rechbauerstraße 12/II, A-8010 Graz

Projektleiter: Univ.-Prof. Dr.techn. Werner GOBIET

Projektpartner:

INSTITUT FÜR ZIVILRECHT (IZR)

Universität Innsbruck

HERRY CONSULT GMBH

Em. Univ.-Prof. Dr. Peter FALLER

\section{VERFASSER: FORSCHUNGSGRUPPE Alp-EmiV}

Univ.-Prof.Dr.techn. Werner GOBIET (ISV, Projektleiter)

Em.Univ.-Prof.Dr. Peter FALLER

Dr.techn. Markus FREWEIN (ISV, Projektmanagement)

Ass.-Prof. Mag. Dr. Peter JORDAN (IZR)

Dr. Max HERRY (Herry Consult Gmbh)

unter Mitarbeit von

Martin VILHAR (ISV)

ISBN-10 3-7001-3778-8

ISBN-13 978-3-7001-3778-8

doi: 10.1553/alp-emiv

http://epub.oeaw.ac.at/alp-emiv

Graz, im Juni 2006 


\section{Anhang}

\subsection{Projektteam Kontakt}

10.1.1 Institut für Straßen- und Verkehrswesen (ISV), TU-Graz

Adresse: Rechbauerstraße 12/II, $8010 \mathrm{Graz}$

Telefon: +43-316-873-6221

Fax: +43-316-873-4199

Email: werner.gobiet@tugraz.at, markus.frewein@verkehrplus.at, isv@tugraz.at Internet: http://www.isv.tugraz.at

Ansprechpartner:

- Univ.-Prof. DI Dr.techn. Werner Gobiet: Projektleiter

- Univ.-Ass. DI Dr.techn. Markus Frewein: Projektmanagement

- Mitarbeiter auf Werkvertragsbasis: Alexander Salamon, Robert WeiBensteiner, DI Martin Vilhar

\subsubsection{Herry Consult GmbH}

Adresse: Argentinierstraße 21, 1050 Wien

Telefon: +43-1-504 1258

Fax: +43-1-504 3536

Email: office@herry.at Internet: http://www.herry.at Ansprechpartner:

- Dr. Max Herry

10.1.3 Institut für Zivilrecht (IZR), Universität Innsbruck

Adresse: Innrain 52, Christoph-Probst-Platz, 6020 Innsbruck

Telefon: +43-512-8113

Fax: +43-316-512-2822

Email: peter.jordan@uibk.ac.at

Internet: http://www2.uibk.ac.at/zivilrecht/mitarbeiter/jordan

Ansprechpartner:

- Ass.-Prof. Mag. Dr. Peter Jordan

10.1.4 Em. Univ.-Prof. Dr. Faller

Adresse: Weidengasse 4, 3424 Wolfpassing

Telefon: +43-2242-70 557

Fax: +43-2242-70 533

Email: peter.faller@aon.at

Ansprechpartner:

- Em. Univ.-Prof. Dr. Peter Faller 


\subsection{Forschungsthemen}

Als Forschungsfragen werden die Fragen bezeichnet, die sich im Rahmen einer Forschungsaufgabe ergeben, respektive beantwortet werden. Für das gegenständliche Projekt ergibt sich durch den interdisziplinären Zugang eine Reihe von Fragen, die weit über den Untersuchungsgegenstand hinausgehen und teilweise anderen Fachdisziplinen zuzuordnen sind.

Für das gegenständliche Projekt stellt es sich demnach als zweckmäßig heraus, die gesammelten Probleme in Forschungsthemen zusammenzufassen. Aus der Vielzahl der Forschungsthemen ist ersichtlich, welchen Forschungsbedarf es im Bereich des „zukunftsfähigen und ökologisch verträglichen“ Verkehrs in ökologisch sensiblen Gebieten gibt.

Die Forschungsthemen wurden wie folgt gegliedert:

- Allgemeine grundlegende Forschungsthemen (),

- Gebietsbezogene Forschungsthemen (),

- Infrastruktur- und verkehrsbezogene Forschungsthemen () und das

- Das Lenkungsinstrument betreffende Forschungsthemen, die in

- Entwicklungsbezogene Forschungsthemen () und

- Umsetzungsbezogene Forschungsthemen () unterteilt werden.

\subsubsection{Allgemeine projektbezogene Forschungsthemen}

In den allgemeinen projektbezogenen Forschungsthemen sind grundsätzliche Fragestellungen, die die Rahmenbedingungen, die Grundphilosophie des gegenständlichen Forschungsprojektes und grundsätzliche ökonomische und ökologische Fragen betreffen, zusammengefasst. 
Tabelle 10: Allgemeine projektbezogene Forschungsthemen (Grundlegende Ansatzpunkte)

\begin{tabular}{|c|c|c|c|}
\hline \multicolumn{3}{|r|}{ ALLGEMEINE FORSCHUNGSTHEMEN } & \multirow{2}{*}{ 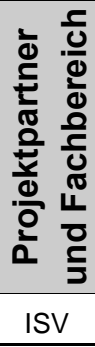 } \\
\hline A-1 & Typen u & eltorientierter Lenkungsinstrumente für Verkehr. & \\
\hline \multicolumn{2}{|c|}{$\begin{array}{l}\text { Erläuterung } \\
\qquad \begin{array}{l}\text { A-1: } \\
\end{array}\end{array}$} & $\begin{array}{l}\text { Eine Zusammenstellung von Verkehrsnachfrage steuernden Maßnah- } \\
\text { men respektive, Lenkungsinstrumenten für Verkehr, soll als Basis für die } \\
\text { Unterscheidung nach möglichen Typen von Lenkungsmechanismen } \\
\text { dienen. Besonderes Augenmerk wird für dieses Forschungsprojekt auf } \\
\text { die „ökologischen Typen“ von Lenkungsinstrumenten geworfen. }\end{array}$ & $\begin{array}{l}\text { VP, } \\
\text { VÖK }\end{array}$ \\
\hline A-2 & \multicolumn{2}{|c|}{$\begin{array}{l}\text { Auswahl der Grundphilosophie für das zu entwickelnde Lenkungsin- } \\
\text { strumentarium: Ist das Prinzip „cap and trade“ der geeignete Ansatz? }\end{array}$} & $\mathrm{Fa}$ \\
\hline \multicolumn{2}{|c|}{$\begin{array}{l}\text { Erläuterung } \\
\qquad \text { A-2: }\end{array}$} & $\begin{array}{l}\text { Diese Frage stellt sich vor allem im Hinblick auf das auf EU-Ebene } \\
\text { beschlossene Ziel einer Querfinanzierung. Können durch den Emissi- } \\
\text { onsrechtehandel die für die Querfinanzierung der Schiene benötigten } \\
\text { Mittel aufgebracht werden? }\end{array}$ & VÖN \\
\hline A-3 & \multicolumn{2}{|c|}{$\begin{array}{l}\text { Lenkungsinstrumentarien im marktwirtschaftlichen Kontext I } \\
\text { Denkansatz: Wie kommt es zum Ausgleich zwischen den Be- } \\
\text { dürfnissen der Wirtschaft, der Gesellschaft und der Umwelt? }\end{array}$} & $\mathrm{Fa}$ \\
\hline \multicolumn{2}{|c|}{$\begin{array}{l}\text { Erläuterung } \\
\text { A-3: }\end{array}$} & $\begin{array}{l}\text { Nachhaltige Entwicklung kann nur stattfinden, wenn alle } 3 \text { Bereiche in } \\
\text { einem ausgewogenen Verhältnis zueinander gefördert werden. }\end{array}$ & VÖN \\
\hline A-4 & \multicolumn{2}{|c|}{ Verständnis der Straße als Emissionen induzierende Anlage. } & IZR \\
\hline \multicolumn{2}{|c|}{$\begin{array}{l}\text { Erläuterung } \\
\qquad A-4 \text { : }\end{array}$} & $\begin{array}{l}\text { Während - im Gefolge technischer Verbesserungen am Fahrzeug Emis- } \\
\text { sionsgrenzwerte für das einzelne Fahrzeuge (Lärm, Schadstoffe) laufend } \\
\text { gesenkt werden können, gibt es - wegen der derzeit unbegrenzten } \\
\text { Freigabe zum Gemeingebrauch - keine rechtliche Möglichkeit, die Ge- } \\
\text { samtemissionen des Verkehrs an einem hoch belasteten Straßenab- } \\
\text { schnitt zu einer bestimmten Zeit an einem bestimmten Ort - insbesonde- } \\
\text { re in sensiblen Gebieten - systematisch unterhalb eines gesundheitli- } \\
\text { chen und ökologischen Grenzwertes zu halten. } \\
\text { Erst eine Betrachtung der Straße als emittierende Anlage, deren Ge- } \\
\text { samtemissionen aller Fahrzeuge (Lärm und Schadstoffe; LKW und } \\
\text { PKW) zu einer bestimmten Zeit an einem bestimmten Straßenabschnitt } \\
\text { Grenzwerten unterliegen, kann die Prinzipien und Erfahrungen des und } \\
\text { historisch erfolgreichen Umweltrechts der Industrie nützen. }\end{array}$ & RT \\
\hline A-5 & \multicolumn{2}{|c|}{$\begin{array}{l}\text { Umsetzung und praktikable Anwendung eines Lenkungsinstru- } \\
\text { ments für den Straßengüterverkehr in ökologisch sensiblen Ge- } \\
\text { bieten. Die Einbindung der Sozialverträglichkeit und Umweltver- } \\
\text { träglichkeit als wesentliche Rahmenbedingung. }\end{array}$} & ISV \\
\hline & $\begin{array}{l}\text { läuterung } \\
\text { A-5: }\end{array}$ & $\begin{array}{l}\text { Umweltverträglichkeit wird durch die Unterstützung einer dauerhaft } \\
\text { tragfähigen Entwicklung der betrachteten Ökosysteme erreicht. Damit } \\
\text { Umweltverträglichkeit erreicht werden kann, kann es sein, dass der } \\
\text { Widerstand im Verkehrssystem (Streckenlänge, Fahrtzeit, Kosten) so } \\
\text { stark zu erhöhen ist, dass es zu einer mehrheitlichen gesellschaftlichen } \\
\text { Ablehnung der Maßnahme (des Lenkungsinstrumentariums) kommen } \\
\text { kann, bzw. die Akzeptanz gering wird. Allerdings ist gerade eine geringe } \\
\text { Akzeptanz die Basis für eine erreichbare Verhaltensänderung im Stra- } \\
\text { Bengüterverkehr - das Akzeptanzdilemma. Populär formuliert: Steigen } \\
\text { die Kosten, steigt die Umweltverträglichkeit, ist das aber sozial verträg- } \\
\text { lich? }\end{array}$ & $\begin{array}{l}\text { VÖK, } \\
\text { VP }\end{array}$ \\
\hline
\end{tabular}




\begin{tabular}{|c|c|c|c|}
\hline & \multicolumn{2}{|c|}{$\begin{array}{l}\text { Fortsetzung Fehler! Verweisquelle konnte nicht gefunden } \\
\text { werden. }\end{array}$} & \\
\hline A- 6 & \multicolumn{2}{|c|}{$\begin{array}{l}\text { Unterschiede bei den Lenkungsinstrumentarien (z.B. Emissions- } \\
\text { zertifikate, Road Pricing, Fixmauten etc.) in Bezug auf Internali- } \\
\text { sierung der externen Kosten. }\end{array}$} & $\mathrm{He}$ \\
\hline \multicolumn{2}{|c|}{$\begin{array}{l}\text { Erläuterung } \\
\text { A-6: }\end{array}$} & $\begin{array}{l}\text { Die Unterschiede bei den Lenkungsinstrumentarien in Bezug auf Interna- } \\
\text { lisierung der externen Kosten sind erheblich und werden in der For- } \\
\text { schungsarbeit herausgearbeitet. }\end{array}$ & VÖN \\
\hline A-7 & \multicolumn{2}{|c|}{$\begin{array}{l}\text { Entwicklung und Begründung eines problemadäquaten ZKI- } \\
\text { Systems (Ziele- Kriterien- Indikatoren- System) für das gegen- } \\
\text { ständliche Forschungsprojekt. }\end{array}$} & ISV \\
\hline \multicolumn{2}{|c|}{$\begin{array}{l}\text { Erläuterung } \\
\qquad \text { A-7: }\end{array}$} & $\begin{array}{l}\text { Es sind die Zusammenhänge zwischen den Ursachen und den Wirkun- } \\
\text { gen bei verkehrsbedingten Umweltproblemen zu erfassen und durch } \\
\text { geeignete Beschreibungsgrößen im Sinne eines „pars-pro-toto“ für die } \\
\text { Erfassung und Evaluierung darzustellen. Für ein ZKI-System, welches } \\
\text { möglichst umfassend die Situation beschreiben, aber auch zur Evaluie- } \\
\text { rung von Maßnahmen dienen soll, sind folgende Fragen relevant: } \\
\text { - Welche Art von Zielen hat als Ausgangspunkt für die Betrachtun- } \\
\text { gen Bedeutung? } \\
\text { - Von welchem Grad an gesellschaftlicher Akzeptanz (Politische } \\
\text { und Nutzer-Akzeptanz) kann bei diesem ZKI-System ausgegangen bzw. } \\
\text { kann damit erreicht werden? } \\
\text { Mit welchen Indikatoren wird die Eignung der vorgeschlagenen } \\
\text { Maßnahmen überprüft? }\end{array}$ & \\
\hline A-8 & \multicolumn{2}{|c|}{$\begin{array}{l}\text { Eignungskonkurrenz der Lenkungsinstrumentarien. Relevanz } \\
\text { von Maßnahmenkonkurrenzen im Rahmen des Forschungspro- } \\
\text { jektes und deren Behandlung. }\end{array}$} & $\mathrm{Fa}$ \\
\hline & $\begin{array}{l}\text { uterung } \\
\text { A-8: }\end{array}$ & $\begin{array}{l}\text { Es muss damit gerechnet werden, dass bestimmte Lenkungsinstrumente } \\
\text { sich gegenseitig unterstützen können, eventuell aber auch sich gegen- } \\
\text { seitig ersetzen können. Dieser Aspekt ist beim vorliegenden Projekt vor } \\
\text { allem deshalb bedeutsam, weil zwei Maßnahmenschwerpunkte (Anla- } \\
\text { genrechtliche Innovationen, Emissionsrechtehandel) untersucht werden. }\end{array}$ & VÖN \\
\hline
\end{tabular}

\subsubsection{Gebietsbezogene Forschungsthemen}

In den gebietsbezogenen Forschungsthemen sind die Themen, die als Grundlage für die Diskussion von ökologisch sensiblen Gebieten dienen, zusammengefasst. 
Tabelle 11: Gebietsbezogene Forschungsthemen

\begin{tabular}{|c|c|c|c|}
\hline \multicolumn{3}{|c|}{ GEBIETSBEZOGENE FORSCHUNGSTHEMEN } & 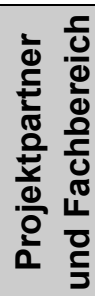 \\
\hline G-1 & \multicolumn{2}{|c|}{$\begin{array}{l}\text { Ökologisch sensible Gebiete im Kontext mit dem Straßengüter- } \\
\text { verkehr. }\end{array}$} & ISV \\
\hline \multicolumn{2}{|c|}{$\begin{array}{l}\text { Erläuterung } \\
\qquad \text { G-1: }\end{array}$} & $\begin{array}{l}\text { Was ökologisch sensibel ist, ist einerseits eine Frage von gesellschaftli- } \\
\text { cher Wertvorstellung und andererseits Ergebnis von wirtschaftlichen } \\
\text { Zwängen (Notwendigkeiten): wenn nämlich das „Erhalten“ von Ökosys- } \\
\text { temen auf der einen Seite und der Straßengüterverkehr als Ergebnis von } \\
\text { wirtschaftlichen Nutzungen auf der anderen Seite im Widerspruch zuein- } \\
\text { ander stehen. Der Straßengüterverkehr mit seinen negativen Auswir- } \\
\text { kungen ist als Störfaktor in ösib-Gebieten zu sehen und somit ist er } \\
\text { kritisch mit den Gebietseigenschaften zu diskutieren. }\end{array}$ & VÖK \\
\hline G-2 & \multicolumn{2}{|c|}{$\begin{array}{l}\text { Adaptierung des allgemeinen ZKI-Systems zur Berücksichtigung der } \\
\text { regionalspezifischen Einflussfaktoren von ösib-Gebieten im Kontext mit } \\
\text { dem Straßengüterverkehr. } \\
\text { (Forschungsthema A-7) }\end{array}$} & ISV \\
\hline \multicolumn{2}{|c|}{$\begin{array}{l}\text { Erläuterung } \\
\qquad \text { G-2: }\end{array}$} & $\begin{array}{l}\text { Das ZKI-System soll einerseits allgemeine Aussagen und Einstufungen } \\
\text { im System Verkehr-Umwelt ermöglichen, andererseits aber regionale } \\
\text { Besonderheiten berücksichtigen. Ein Bündel von ausgewählten Kriterien } \\
\text { und Indikatoren dient zur Abschätzung der allgemeinen Trendentwick- } \\
\text { lungen, eine geeignete Erweiterung zur Darstellung und Berücksichti- } \\
\text { gung der regionalspezifischen Besonderheiten des betrachteten Pla- } \\
\text { nungsgebietes erfolgt unter Berücksichtigung der Aussagen von For- } \\
\text { schungsthema G-1. }\end{array}$ & $\begin{array}{l}\text { VP, } \\
\text { VÖK }\end{array}$ \\
\hline G-3 & \multicolumn{2}{|c|}{$\begin{array}{l}\text { Feststellung und Diskussion der Überlastungsgrenzen eines ökolo- } \\
\text { gisch sensiblen Gebietes und eine darauf fundierte Einstufung dieser } \\
\text { Gebiete. }\end{array}$} & ISV \\
\hline \multicolumn{2}{|c|}{$\begin{array}{l}\text { Erläuterung } \\
\qquad \text { G-3: }\end{array}$} & $\begin{array}{l}\text { Der Funktionsfähigkeit eines Gebietes als Ergebnis der ökologischen } \\
\text { Zusammenhänge steht die Verkehrsnotwendigkeit als Funktion der } \\
\text { menschlichen Nutzungen mit all ihren negativen Auswirkungen auf die } \\
\text { Umwelt gegenüber. Es sind also die Überlastungsgrenzen für Ökologie } \\
\text { als auch von Ökonomie (im Sinne der realisierten Nutzungen im Gebiet) } \\
\text { zu diskutieren und als Basis einer Einstufung der Gebiete „vergleichbar“ } \\
\text { zu machen. }\end{array}$ & VÖK \\
\hline G-4 & \multicolumn{2}{|c|}{$\begin{array}{l}\text { Emissions- und Immissionsverhältnisse: Alpentäler, Mittelgebirgstäler } \\
\text { und Flachland im Vergleich. }\end{array}$} & ISV \\
\hline \multicolumn{2}{|c|}{$\begin{array}{l}\text { Erläuterung } \\
\qquad \text { G-4: }\end{array}$} & $\begin{array}{l}\text { Die Topographie und die meteorologischen Verhältnisse eines Gebietes } \\
\text { können die negativen Wirkungen durch Schadstoffe verstärken oder } \\
\text { auch abschwächen. Für das gegenständliche Lenkungsinstrumentarium } \\
\text { ist eine detaillierte Diskussion der gebietsbedingten Einflussfaktoren } \\
\text { wesentlich und zu berücksichtigen. }\end{array}$ & VÖK \\
\hline G-5 & \multicolumn{2}{|c|}{$\begin{array}{l}\text { Projektion der Umweltauswirkungen: Schadstoff- und Lärmbelastung } \\
\text { durch Straßenverkehr bei Fortdauer der bisherigen Verkehrsentwick- } \\
\text { lung im Jahr } 2020 \text { ( } 2030,2050) \text {. } \\
\text { Welcher Beitrag kann durch die Entwicklung der Technologie erwartet } \\
\text { werden? }\end{array}$} & $\mathrm{He}$ \\
\hline
\end{tabular}




\begin{tabular}{|c|c|c|c|}
\hline \multicolumn{2}{|c|}{$\begin{array}{c}\text { Erläuterung } \\
\qquad \text { G-5: }\end{array}$} & $\begin{array}{l}\text { Es wird eine zukünftige Entwicklung der Schadstoff- und Lärmbelastung } \\
\text { durch Straßenverkehr bei Fortdauer der bisherigen Verkehrsentwicklung } \\
\text { im Jahr } 2020 \text { (2030, 2050) und der in dieser Arbeit getroffenen Maß- } \\
\text { nahmen erarbeitet. } \\
\text { Außerdem wird der Beitrag der Technologie in der gegenständlichen } \\
\text { Arbeit durch die darin enthaltenden Maßnahmen abgeschätzt. Dieser } \\
\text { Beitrag wird erheblich sein, aber allein das Umweltproblem nicht lösen } \\
\text { können. }\end{array}$ & VP \\
\hline G-6 & \multicolumn{2}{|c|}{$\begin{array}{l}\text { Projektion der Umweltauswirkungen: Voraussichtlicher Vorteil der an- } \\
\text { lagenrechtlichen Lenkungsinstrumente bei der Entwicklung der Schad- } \\
\text { stoff- und Lärmbelastung in ökologisch sensiblen Gebieten. }\end{array}$} & IZR \\
\hline & $\begin{array}{l}\text { läuterung } \\
\text { G-6: }\end{array}$ & $\begin{array}{l}\text { Um die Immissionen in der Umgebung der Straßenverkehrsanlage zu } \\
\text { stabilisieren und schrittweise zu senken, ist es notwendig, die Gesamt- } \\
\text { emissionen der Anlage (an dem Straßenabschnitt) - wie jene der Indust- } \\
\text { rie - rechtsverbindlichen Grenzwerten zu unterwerfen. Gleichzeitig sind } \\
\text { dem Anlagenbetreiber jene Mittel in die Hand zu geben, den Verkehrs- } \\
\text { prozess so zu steuern, dass die Gesamtemission unterhalb des (Ge- } \\
\text { samt)Grenzwertes für die Anlage gehalten wird. Dazu stehen ihm } \\
\text { grundsätzlich die Mittel der sog. Straßenpolizei zur Verfügung: Fahrver- } \\
\text { bote, Geschwindigkeitsbeschränkungen, Fahrstreifenverengung oder - } \\
\text { freigabe }\end{array}$ & RT \\
\hline
\end{tabular}

\subsubsection{Infrastrukturbezogene Forschungsthemen}

In den Infrastrukturbezogenen Forschungsthemen sind die Themen, die in direktem Zusammenhang mit verkehrlicher Infrastruktur stehen, zusammengefasst.

Tabelle 12: Infrastrukturbezogene Forschungsthemen

\begin{tabular}{|c|c|c|c|}
\hline \multicolumn{3}{|c|}{ INFRASTRUKTURBEZOGENE FORSCHUNGSTHEMEN } & 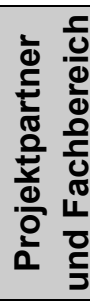 \\
\hline I-1 & \multicolumn{2}{|c|}{$\begin{array}{l}\text { Definition von entsprechenden Straßenabschnitten als Emissio- } \\
\text { nen induzierende Anlage : räumlich, modal, sektoral und tempo- } \\
\text { ral. }\end{array}$} & ISV \\
\hline & $\begin{array}{l}\text { äuterung } \\
\text { I-1: }\end{array}$ & $\begin{array}{l}\text { Die Definition soll Basis für die rechtliche Handhabe als Anlage im Sinne } \\
\text { des Umweltanlagenrechtes bilden. Es sind, neben den Gebieten, die } \\
\text { über ihre naturräumlichen Gegebenheiten als ökologisch sensibel gelten, } \\
\text { wie z.B. der Alpenraum, auch die dichtbesiedelten Gebiete, die von den } \\
\text { negativen Auswirkungen durch Industrie und Verkehr betroffen sind, z.B. } \\
\text { das Ruhrgebiet, zu betrachten und u. U. sogar für das gegenständliche } \\
\text { Forschungsprojekt von Relevanz. }\end{array}$ & VP \\
\hline \multicolumn{3}{|c|}{$\begin{array}{l}\text { Vorteil des Lenkungsbeitrages der anlagenrechtlichen Lenkungsinstrumente, } \\
\text { diskutiert am Beispiel der wesentlichen Determinanten des Straßengüterver- } \\
\text { kehrs: } \\
\text { - Verkehrsleistung } \\
\text { - Verkehrsaufkommen } \\
\text { - Zeitliche und räumliche Verkehrsverlagerung } \\
\text { - Ökoeffizienz der Fahrzeuge (Auslastung, Nettotonnenkilometer) }\end{array}$} & ISV \\
\hline
\end{tabular}




\begin{tabular}{|c|c|c|c|}
\hline \multicolumn{2}{|c|}{$\begin{array}{c}\text { Erläuterung } \\
\qquad I-2:\end{array}$} & $\begin{array}{l}\text { Im Anlagenrecht besteht die Möglichkeit Grenzwerte für z.B. Schad- } \\
\text { stoffsausstoß verbindlich zu machen. Durch diese „Einflussmöglichkeit" } \\
\text { ist eine Steuerung einer (gewünschten) Entwicklung des Straßengüter- } \\
\text { verkehrs gegeben. Die beispielhaft angeführten Determinanten würden } \\
\text { somit in einer Art gesteuert werden können, für die derzeit die rechtliche } \\
\text { Grundlage fehlt. }\end{array}$ & VP \\
\hline I-3 & \multicolumn{2}{|c|}{$\begin{array}{l}\text { Untersuchungsrelevante Lösungspotenziale im Straßengüterverkehr } \\
\text { durch die anlagenrechtlichen Lenkungsinstrumente (z.B. Rationali- } \\
\text { sierungspotenziale). }\end{array}$} & $\mathrm{He}$ \\
\hline & $\begin{array}{l}\text { äuterung } \\
\text { I-3: }\end{array}$ & $\begin{array}{l}\text { Wie wirkt das anlagenrechtliche Lenkungsinstrumentarium z.B. auf das } \\
\text { Durchschnittsalter der LKW-Flotte etc oder auf deren technologische } \\
\text { Entwicklung (Prinzip der Best Available Technique). Ist eine Wirkung der } \\
\text { ökologisch motivierten Abgabendifferenzierung auf das Investitionsver- } \\
\text { halten der LKW-Betreiber zu erwarten? }\end{array}$ & $\begin{array}{l}\text { VP, } \\
\text { VÖN }\end{array}$ \\
\hline
\end{tabular}

\subsubsection{Lenkungsinstrumentbezogene Forschungsthemen}

\subsubsection{Entwicklungsbezogene Forschungsthemen}

Die entwicklungsbezogenen Forschungsthemen betreffen die Entwicklung und wissenschaftliche Absicherung des gegenständlichen Lenkungsinstrumentariums. Es sind dies die Themen, die den theoretischen Rahmen für eine spätere realistische Umsetzung definieren.

Tabelle 13: Lenkungsinstrument: Entwicklungsbezogene Forschungsthemen

\begin{tabular}{|c|c|c|c|}
\hline \multicolumn{3}{|c|}{$\begin{array}{l}\text { LENKUNGSINSTRUMENT: } \\
\text { ENTWICKLUNGSBEZOGENE FORSCHUNGSTHEMEN }\end{array}$} & 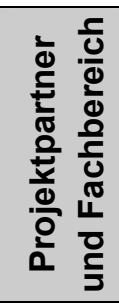 \\
\hline LE-1 & $\begin{array}{l}\text { Welche } \\
\text { Emissi }\end{array}$ & $\begin{array}{l}\text { Indlagen und Voraussetzungen müssen für den Handel mit } \\
\text { ertifikaten im Verkehr erfüllt sein? }\end{array}$ & $\mathrm{Fa}$ \\
\hline \multicolumn{2}{|c|}{$\begin{array}{l}\text { Erläuterung } \\
\qquad L E-1:\end{array}$} & $\begin{array}{l}\text { - Errechnung der Gesamtzahl an Zertifikaten, die notwendig sein } \\
\text { werden, um das ökologisch begründbare „Einfrieren“ der Transportmen- } \\
\text { ge auf einer sensiblen Strecke zu bewirken. } \\
\text { - Verteilungsplan für die Zertifikate an die bisherigen Nutzer der } \\
\text { Strecke: Wann, wer und wie viel? } \\
\text { - Reservehaltung (Zertifikate für hinzukommende Nutzer) } \\
\text { - Zeitliche Gültigkeit der Zertifikate? } \\
\text { - Wie werden die Zertifikate gebucht? (Ähnlich Flugbuchungssys- } \\
\text { teme, Börse für Durchfahrtsrechte) } \\
\text { - Prognose des voraussichtlichen Nachfrageüberhanges und des } \\
\text { daraus zu erwartenden Handelswertes der Zertifikate. } \\
\text { - Notwendigkeit und Aufgaben eines Monitoring Systems: } \\
\text { - Laufende Beobachtung der Marktpreise der Zertifikate } \\
\text { - Laufende Beobachtung der Struktur der im sensiblen Gebiet } \\
\text { weiterhin transportierten Güter } \\
\text { - Laufende Beobachtung der Marktanteile der Nutzer im sensiblen } \\
\text { Gebiet }\end{array}$ & VÖN \\
\hline \multicolumn{3}{|c|}{$\begin{array}{l}\text { Geeignete Indikatoren der Umweltbelastung (Schadstoffemission, } \\
\text { Fahrleistung, Lärmemission etc.) als Grundlage für die anlagen- } \\
\text { rechtliche Verkehrssteuerung. }\end{array}$} & IZR, ISV \\
\hline
\end{tabular}




\begin{tabular}{|c|l|l|l|}
\hline \multicolumn{2}{|c|}{$\begin{array}{c}\text { Erläuterung } \\
\text { LE-2: }\end{array}$} & $\begin{array}{l}\text { Diese Indikatoren sind Teil des Ziele-Kriterien-Indikatoren Systems und } \\
\text { werden im Zuge des Forschungsprojektes interdisziplinär abgestimmt. }\end{array}$ & RT \\
\hline LE-3 & $\begin{array}{l}\text { Bestimmung von Grenzwerten für bestimmte Gebiete. } \\
\text { Prinzip der Subsidiarität: Wer setzt diese Werte fest: Nationale } \\
\text { und/oder internationale Behörden? }\end{array}$ & ISV \\
\hline \multirow{2}{*}{$\begin{array}{l}\text { Erläuterung } \\
\text { LEl-3: }\end{array}$} & $\begin{array}{l}\text { Internationale Grenzwerte der WHO, Grenzwerte der EU, in der umwelt- } \\
\text { bezogenen Forschung der Österreichischen Akademie der Wissenschaf- } \\
\text { tentwickelte Grenzwerte für luftverunreinigende Substanzen, (steuer-) } \\
\text { Grenzwerte zur Beeinflussung des Investitions- und Fahrverhaltens der } \\
\text { Nutzer der Anlage. Die Bestimmung von Grenzwerten ist eine wesentli- } \\
\text { che Aufgabe des Forschungsprojektes. Zentrale Anforderung ist die } \\
\text { wissenschaftliche Absicherung der Grenzwerte für ökologisch sensible } \\
\text { Gebiete im Kontext mit dem Straßengüterverkehr. }\end{array}$ & VÖK \\
\hline
\end{tabular}

Die weiteren Forschungsthemen befinden sich noch in Diskussion. Es sind dies drei Forschungsthemen, die die Entwicklung des Lenkungsinstrumentes, und alle Forschungsthemen die die Umsetzung des Lenkungsinstrumentes betreffen.

Tabelle 14: Lenkungsinstrument: Entwicklungsbezogene Forschungsthemen, die sich noch in Diskussion befinden

\begin{tabular}{|c|c|c|}
\hline & $\begin{array}{c}\text { LENKUNGSINSTRUMENT: } \\
\text { ENTWICKLUNGSBEZOGENE FORSCHUNGSTHEMEN } \\
\text { Diskussionsgrundlage }\end{array}$ & 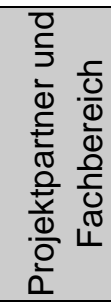 \\
\hline LE-5 & $\begin{array}{l}\text { Welcher Berechnungsdatensatz (Verkehr, Wetterlage, Tageszeit, } \\
\text { Grenzwerte usw.) ist als Eingangsparameter für Modellrechnungen } \\
\text { (anlagenrechtliche Lenkungsinstrumente) notwendig, damit das Über- } \\
\text { schreiten von bestimmten Grenz- bzw. Richtwerten für Emissionen } \\
\text { (Immissionen?) verhindert wird? } \\
\text { Welcher Detaillierungsgrad der Inputdaten ist für eine praktikable E- } \\
\text { missionssteuerung entsprechend der Projektgrundphilosophie notwen- } \\
\text { dig? }\end{array}$ & ISV, $\mathrm{He}$ \\
\hline LE-6 & $\begin{array}{l}\text { Wen belastet eine Senkung von Grenzwerten (Immissionen und Emis- } \\
\text { sionen), wer hat Vorteile dadurch? }\end{array}$ & $\mathrm{Fa}, \mathrm{He}$ \\
\hline LE-7 & $\begin{array}{l}\text { Intermodalität des Lenkungsinstrumentes (2. Jahr): Ist die Er- } \\
\text { mittlung und Verankerung von Emissionskorridoren möglich und } \\
\text { vertretbar (Gebietsfestlegung, gesetzliche Verankerung)? } \\
\text { Definition Emissionskorridor: Es wird die Summe aller Emissionen aller Verkehrsmittel } \\
\text { auf den vorhandenen Verkehrsträgern in einem definierten Verkehrskorridor addiert und } \\
\text { als Obergrenze festgelegt. (Grenzwerte der Emissionen: Abhängigkeit bzw. deren Her- } \\
\text { leitung ist zu klären.) }\end{array}$ & IZR \\
\hline
\end{tabular}

\subsubsection{Umsetzungsbezogene Forschungsthemen}

Die umsetzungsbezogenen Forschungsthemen betreffen die Umsetzung des gegenständlichen Lenkungsinstrumentariums. Es sind dies die Themen, die als 
Basis für eine praktikable Umsetzung behandelt werden müssen. Die unten zusammengefassten Forschungsthemen befinden sich noch in interdisziplinärer fachlicher Diskussion.

Tabelle 15: Lenkungsinstrument: Umsetzungsbezogene Forschungsthemen, die sich noch in Diskussion befinden

\begin{tabular}{|l|l|c|}
\hline \multicolumn{1}{|c|}{$\begin{array}{l}\text { LENKUNGSINSTRUMENT } \\
\text { Diskussionsgrundlage }\end{array}$} & $\begin{array}{c}\text { UMSETZUNGSBEZOGENE FORSCHUNGSTHEMEN } \\
\text { LU- }\end{array}$ & $\begin{array}{l}\text { Betrieb des Straßenabschnittes: Kann auf Grund von Kurzzeit- } \\
\text { prognosen in den Zulaufstrecken eine Überschreitung der Emissions- } \\
\text { grenzwerte vorausgesagt und so gewährleistet werden, dass } \\
\text { rechtzeitig Maßnahmen ergriffen werden können, dass es zu keiner } \\
\text { bzw. zu einer geringfügigen Überschreitung und zu keiner } \\
\text { wesentlichen Störung des Verkehrsablaufes im Güterverkehr kommt? }\end{array}$ \\
\hline LU-2 & $\begin{array}{l}\text { Für den Fall, dass auch Immissionen im Lenkungsinstrument Basis für } \\
\text { eine Situationsbeurteilung (Grenzwertüberschreitung) sind: Wie wird } \\
\text { ein Verhältnis zwischen Emissionen des Verkehrs auf dem betrachte- } \\
\text { ten Straßenabschnitt und den vorhandenen gemessenen Immissions- } \\
\text { belastungen (teilweise andere Ursachen) hergestellt? }\end{array}$ & Alle \\
\hline LU-3 & $\begin{array}{l}\text { Wie kann verhindert werden, dass falsche Angaben der Fahrzeug- } \\
\text { betreiber über Emissionswerte die Steuerung der Anlage verfälschen? } \\
\text { (Kontrolle der technischen Ausstattung der Fahrzeuge, Preisberech- } \\
\text { nung für die Emissionszertifikate) }\end{array}$ & ISV, He \\
\hline LU-4 & $\begin{array}{l}\text { Sind die vorgeschlagenen Maßnahmen innerhalb einer realisti- } \\
\text { schen Zeitspanne (mittel- bis langfristig) umsetzbar? }\end{array}$ & Fa, He \\
\hline
\end{tabular}

\title{
10.3 Überblick über Typen umweltorientierter Lenkungs- instrumente für Straßengüterverkehr
}

\author{
Markus FREWEIN, Werner GOBIET, Alexander SALAMON
}

In diesem Kapitel werden die Bezeichnungen Lenkungsmechanismen, Lenkungsinstrumente und Lenkungsmaßnahmen in eine begründbare Ordnung gebracht. Es wird für die umweltorientierte und umweltschonende Abwicklung des Verkehrs eine Systematik aufgestellt und verschiedenen Umfeldbereichen entsprechende Maßnahmen zugeordnet. Aufbauend auf die Erklärung der schematischen Einteilung werden im einzelnen Lenkungsmechanismen und Lenkungsinstrumente. Bei den Lenkungsinstrumenten erfolgt der Fokus auf die umweltorientierten Instrumente, die nach Ordnungs- und Marktpolitischen Instrumenten geordnet werden. Abschließend erfolgt in einer Kombinationsmatrix eine Zuordnung 
von Maßnahmen zu Lenkungsmechanismen und umweltorientierten Lenkungsinstrumenten.

\subsubsection{Unterscheidung von Begriffen für die Verkehrslenkung}

Wie in Abbildung 27 ersichtlich ist, empfiehlt es sich zwischen Lenkungsmechanismen und Lenkungsinstrumenten zu unterscheiden. Lenkungsmechanismen umfassen somit die Lenkungsinstrumente und die möglichen Maßnahmen bzw. Maßnahmenbündel für verschiedene Umfeld- und Wirkungsbereiche.

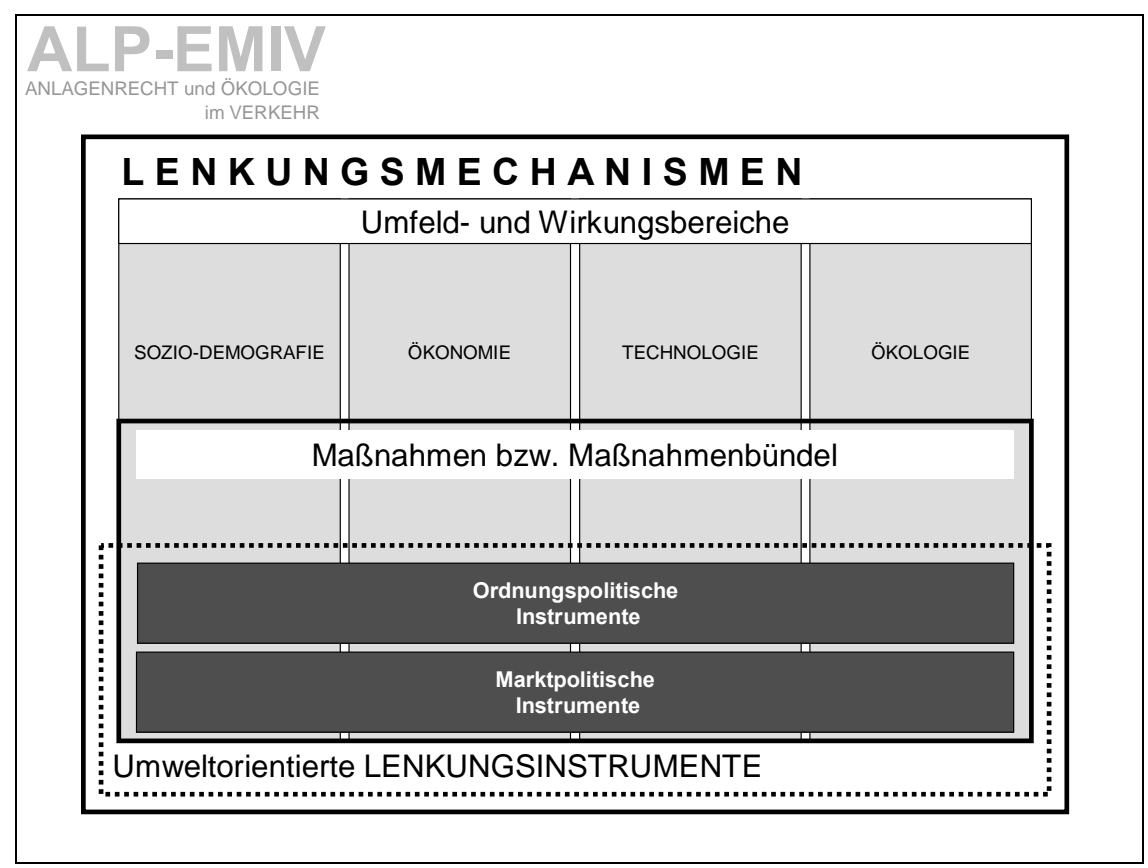

Abbildung 27: Prinzipielle Ordnung zwischen Lenkungsmechanismen und Lenkungsinstrumenten mit Fokus auf umweltorientierte Lenkungsinstrumente.

Es ist auch in Abbildung 27 ersichtlich, dass eine Teilmenge der Maßnahmen den umweltorientierten Lenkungsinstrumenten zuzuordnen ist.

\subsubsection{Lenkungsmechanismen}

Lenkungsmechanismen im Sinne der Verkehrsachfragelenkung werden wie folgt definiert: Aufbauend auf Maßnahmen und Maßnahmenbündeln sowie Lenkungsinstrumentarien, welche Werkzeuge zur Beeinflussung der Verkehrsnachfrage darstellen, sind Lenkungsmechanismen als übergeordnete Zielvorstellungen anzusehen, die mit Hilfe der Maßnahmen respektive Instrumentarien erreicht werden sollen. Das heißt Lenkungsmechanismen sind nach dieser Definition zielund motivationsorientiert. Es ist damit nun möglich jeder Einzelmaßnahme über die Zuordnung durch Instrumentarien verknüpft mit der dahinterliegenden Motivation, einem Typ von Lenkungsmechanismus in Bezug auf Verkehrsnachfragelenkung zuzuordnen. 
Als Grundlage der Überlegungen für eine neue Systematik diente Cerwenka (Cerwenka et al. 2000) mit der Zusammenstellung der Wechselwirkung zwischen Raumnutzung und Raumüberwindung. Sie zeigt recht anschaulich die Beziehungen zwischen den einzelnen Umfeldern, in denen Verkehr eingebettet ist.

Aus diesen Umfeldbereichen des Verkehrs in Verbindung mit den dahinterliegenden Motivationen wurde nun eine Einteilung erstellt, die sich auf vier große Bereiche aufteilt und die als übergeordnete Systematik dienen soll (vgl. Tabelle 16). Die vier Typen von Lenkungsmechanismen in der Verkehrsnachfragelenkung lauten:

- Sozio-Demografischer Lenkungsmechanismus,

- Ökonomischer Lenkungsmechanismus,

- Technologischer Lenkungsmechanismus und

- Ökologischer Lenkungsmechanismus.

Den Gesellschaftsbereichen, die auch für die Typenbezeichnung für die Lenkungsmechanismen verantwortlich sind, können Umfeldbereiche zugeordnet werden. Bei den Umfeldbereichen ist eine Redundanz zwischen den Gesellschaftsbereichen möglich. Es ist nicht trennscharf zwischen den einzelnen Bereichen zu unterscheiden, außerdem ergibt sich dafür auch keine Notwendigkeit. Im Folgenden werden die einzelnen Typen von Lenkungsmechanismen detailliert erläutert. 
Tabelle 16: Verkehrslenkungsmechanismen unterteilt nach Gesellschafts- und Umfeldbereichen (nach Cerwenka et al. 2000, stark erweitert)

\begin{tabular}{|c|c|c|}
\hline GESELLSCHAFTSBEREICH & UMFELDBEREICH & $\begin{array}{l}\text { ZUGEHÖRIGE MASS- } \\
\text { NAHMEN (AUSWAHL) }\end{array}$ \\
\hline \multirow{3}{*}{ SOZIO-DEMOGRAFIE } & Soziale Prozesse & \multirow{3}{*}{$\begin{array}{l}\text { - Veränderung des Ord- } \\
\text { nungsrahmens } \\
\text { - Bewusstseinsbildung } \\
\text { - Abstimmung der Verkehrs- } \\
\text { und Siedlungsplanung }\end{array}$} \\
\hline & Siedlungsstruktur & \\
\hline & Bevölkerungsentwicklung & \\
\hline \multirow{6}{*}{ ÖKONOMIE } & Wirtschaft und Gesellschaft & \multirow{6}{*}{$\begin{array}{l}\text { - Fahrtenkontingentierung } \\
\text { - CO2-Abgabe } \\
\text { - Treibstoffzoll } \\
\text { - Road Pricing }\end{array}$} \\
\hline & Alternative Verkehrsträger & \\
\hline & Technologie & \\
\hline & Infrastruktur & \\
\hline & Logistik & \\
\hline & Restriktionen & \\
\hline \multirow{3}{*}{ TECHNOLOGIE } & Technologische Entwicklung & \multirow{3}{*}{$\begin{array}{l}\text { - Emissionsgrenzwerte für } \\
\text { Fahrzeuge } \\
\text { - Vermeidung von Straßen- } \\
\text { bau } \\
\text { - Gewichtsbeschränkungen }\end{array}$} \\
\hline & Verkehrssicherheit & \\
\hline & Energie & \\
\hline \multirow{7}{*}{ ÖKOLOGIE } & Raumordnung & \multirow{7}{*}{$\begin{array}{l}\text { - Zeitliche, sektorale und/oder } \\
\text { lokale Fahrverbote } \\
\text { - Verkehrsvermeidung } \\
\text { - Öko-Bonus } \\
\text { - Schwerverkehrsabgabe } \\
\text { - Ausweitung des Angebotes } \\
\text { auf der Schiene }\end{array}$} \\
\hline & Infrastruktur & \\
\hline & Logistik & \\
\hline & Fuhrpark & \\
\hline & Verkehrsordnungspolitik & \\
\hline & Preispolitik & \\
\hline & Öffentlichkeitsarbeit & \\
\hline
\end{tabular}

\subsubsection{Soziodemografischer Lenkungsmechanismus}

Dieser Lenkungsmechanismus deckt all jene Bereiche ab, deren Ziel es ist das soziale, stets mit dem Verkehrssystem verbundene Umfeld des Menschen zu verändern bzw. zu beeinflussen. Es sind dies die Umfeldbereiche Soziale Prozesse, Siedlungsstruktur und Bevölkerung.

\section{- Soziale Prozesse:}

Soziale Prozesse stellen eine grundlegende, vorgelagerte Ebene von Bestimmungsfaktoren für zukünftige (auch verkehrsrelevante) Entwicklungen dar, wobei Wertvorstellungen, Verhaltensweisen und Zeitnutzungsstrukturen eine große Rolle spielen, die wiederum stark von technologischen Entwicklungen geprägt sind (vgl. Cerwenka et al. 2000, Seite 54). Davon wirken folgende soziale Prozesse unmittelbar auf das Verkehrsgeschehen ein: soziale Beziehungen und Beteiligungen, Zeitverwendung und Rollenverhalten. 


\section{- Siedlungsstruktur:}

Als Siedlungsstruktur wird die räumliche Verteilung von Bevölkerung, Arbeitsstätten, etc. bezeichnet. Diese entstehen als Resultat eines Wechselspiels von Wünschen und Möglichkeiten aufgrund von Selbststeuerungsprozessen innerhalb und zwischen Akteursgruppen (Privathaushalte, Unternehmen, öffentliche Stellen) und Rahmenbedingungen der räumlichen Entwicklung mit Rückkoppelung zu sozialen Prozessen, Ressourcen der Umwelt, Energiepreisen und technischem Fortschritt (vgl. Cerwenka et al.2000 S.55).

\section{- Bevölkerungsentwicklung:}

Die Bevölkerungsentwicklung hat auch einen gewissen, wenn auch nicht so großen, Einfluss auf die Menge und Fahrtzweckstruktur des Güterverkehrs. Als eine der Verkehrsnachfrage relevanten Kenngrößen wären die Gesamtbevölkerung, die Bevölkerungsdichte oder auch die Bevölkerungsverteilung zu nennen.

\subsubsection{2 Ökonomischer Lenkungsmechanismus}

Als Umfeldbereiche können dem Gesellschaftsbereich Ökonomie u. a. Wirtschaft und Gesellschaft, Alternative Verkehrsträger, Technologie, Infrastruktur, Logistik und Restriktionen zugeordnet werden.

Im Güterverkehr sind vor allem die Veränderungen in der Produktionsstruktur (und damit in der Transportgüterstruktur) und in der räumlichen Entwicklung der Zuordnung von Produktionsstandorten und Absatzmärkten von Bedeutung. Da bestimmte Transportgüter bestimmte Affinitäten zu bestimmten Gütertransportmitteln haben, hat eine Veränderung der Transportgüterstruktur auch einen Einfluss auf die modale Struktur.

Eine wesentliche wirtschaftliche Einflussgröße im Güterverkehr ist der Anteil der Transportkosten an der gesamten Wertschöpfung des Produktionsprozesses. Dieser Anteil ist in der Vergangenheit tendenziell gesunken (Cerwenka et al. 2000)

Hier setzt nun auch der ökonomische Lenkungsmechanismus an. Durch Veränderung des Verkehrsabgabensystems, Road-pricing und auch der Internalisierung externer Kosten könnte entschieden in die Verkehrsnachfrage in die einzelnen Bereiche (Straße-Schiene) eingegriffen werden. Wobei es hier immer zu beachten gilt: Zum ökonomischen Lenkungsmechanismus werden nur die wirtschaftlich motivierten Bereiche gezählt. Nicht die Art der Werkzeuge ist relevant, sondern das angestrebte Ziel. 


\subsubsection{Technologischer Lenkungsmechanismus}

Diesem Lenkungsmechanismus sind die Umfeldbereiche:

- Technologie,

- Verkehrssicherheit und

- Energie zuzuordnen.

- Technologie:

Technologische Entwicklungen beeinflussen maßgeblich unser gesamtes Handeln in den unterschiedlichsten Bereichen des Lebens. Welcher Art die zu erwartenden Effekte neuer Technologien sind, hängt hauptsächlich von deren Realisierungschancen ab. Diese wiederum sind abhängig von z.B. unterschiedlichen Kriterien, wie z.B.: Marktreife, Rentabilität, Akzeptanz durch Nutzer und immer stärker in den Vordergrund tretend auch Umwelt- und Ressourcenschonung. Zur Abschätzung von Veränderungen in Umfang und Struktur der Verkehrsnachfrage lassen sich drei wichtige Unterbereiche definieren: Verkehrstechnologie, Kommunikationstechnologie und Produktionstechnologie.

\section{- Verkehrssicherheit:}

Die Verkehrssicherheit ist jene Einflussgröße in der Verkehrsnachfrage, die die Abwehr und Minimierung von Gefahren beim Fahren als Anliegen hat. Das gesamte Verkehrssystem (z.B. Infrastruktur, Rollmaterial, Logistik) ist geprägt von Verkehrssicherheitsmaßnahmen. Der entscheidende kritische Faktor ist hier allerdings der Mensch mit seiner Schwäche zu Fehler behaftetem Handeln und dem Missbrauch des Vorgangs der Ortsveränderung zu subjektiv besetzten sozialen Aktivitäten. Hier finden also jene Maßnahmen Eingang, welche zu einer Reduktion des Gefahrenpotenzials bei der Nutzung des gesamten Verkehrssystems dienen und dem Menschen dabei unterstützen, Fehler zu vermeiden oder diese ohne allzu schwere Folgen geschehen zu lassen.

\section{- Energie:}

Die Energieabhängigkeit des Verkehrs ist eine andere massive Einflussgröße. Diese vor allem von fossilen Energieträgern bestehende Abhängigkeit rückt immer wieder in den Vordergrund, einerseits aus Verknappungsgründen, andererseits aus Emissionsgründen. Dieser Bereich ist beeinflusst, wie z.B. von den technologischen Möglichkeiten, den vorhandenen Ressourcen. Hier herein fallen vor allem Maßnahmen zur Verbesserung der Energiebilanz im Verkehr.

\subsubsection{4 Ökologischer Lenkungsmechanismus}

Der ökologische Lenkungsmechanismus umfasst die Umfeldbereiche Raumordnung, Infrastruktur, Logistik, Fuhrpark, Verkehrsordnungspolitik, Preispolitik und Öffentlichkeitsarbeit.

Der Ökologische Lenkungsmechanismus sollte nun Maßnahmenbündel beinhalten, die im Sinne der Umweltschonung motiviert sind. Das heißt, all jene, welche 
eine Verbesserung oder zumindest eine Nicht-Verschlechterung der durch den Verkehr verursachten Umweltsituation herbeiführen. Festzustellen ist, dass die Verkehrsimmissionen eine ständig steigende Umweltbelastung darstellen. Davon betroffen sind alle Bereiche unserer Umwelt, einschließlich des Menschen. Und auch trotz der Bemühungen der letzten Jahre durch den technischen Fortschritt lässt sich eine weitergehende Verschlechterung der Situation vorhersagen. In diesem Sinne ist die Verwirklichung einer umweltschonenden und nachhaltigen Mobilität vor allem durch eine maßgebliche Reduktion der Verkehrsimmissionen möglich (vgl. Kerschner 2001). Um dieses Ziel zu erreichen, können im ökologischen Lenkungsmechanismus, in diesem Sinne Ziel führende Maßnahmen bzw. Maßnahmenbündel dafür zusammengefasst werden. Nachfolgende Zusammenstellung zeigt die Ziele die für Maßnahmen in den Umfeldbereichen gelten sollten (vgl. Kerschner 2001):

- Verkehrsvermeidung,

- Verkehrsverlagerung auf umweltfreundlichere Verkehrsträger,

- Verkehrsorganisation im Sinne der Ökologie,

- Umweltverträglicher Umgang mit Verkehrsflächen,

- Förderung Objekt bezogener technischer Schutzmaßnahmen,

- Förderung umweltverträglicher Fahrzeugkonzepte,

- ökologisch motivierte Preispolitik und

- Öffentlichkeitsarbeit.

Durch ernsthafte und intensive Anwendung der aufgeführten Maßnahmenbereiche oder einer Kombination dieser sollte eine Verbesserung im Sinne der Erhaltung der ökologischen Eigenschaften herbeigeführt werden.

\subsubsection{Was sind Lenkungsinstrumente/-maßnahmen?}

Durch den Gebrauch von Instrumenten erweitern und steigern wir den Nutzen unserer Tätigkeit. Im Folgenden werden der Begriff Lenkungsinstrument und der Begriff Lenkungsmaßnahme synonym verwendet.

Lenkungsinstrumente für Verkehrsnachfragesteuerung im Verkehrswesen sind folglich Bündel von Einzelmaßnahmen, welche gesetzt werden, um die Verkehrsnachfrage in einer gewünschten Art und Weise zu lenken. Diese Verkehrsnachfrage steuernde Maßnahmen lassen sich nach Cerwenka (Cerwenka et al. 2000) folgend unterteilen:

- Raumordungspolitische Maßnahmen

- Verkehrsinfrastrukturelle Maßnahmen

- Logistische Maßnahmen 
- Fahrzeugtechnische Maßnahmen

- Verkehrsordnungspolitische Maßnahmen

- Preispolitische Maßnahmen

Bei Cerwenka/Hauger/Hörl (BMU 2004, Seite 29) gibt es eine ähnliche Systematik der Lenkungsinstrumente bzw. -maßnahmen, und zwar folgende:

- Ordnungspolitische Instrumente

- Marktpolitische Instrumente

- Intrastrukturpolitische Maßnahmen

- Preispolitische Maßnahmen

- Logistische Maßnahmen

Die Einzelmaßnahmen zur umweltorientierten Abwicklung des Verkehrs werden in Maßnahmengruppen und danach in zwei Maßnahmenbereiche - ordnungspolitische und marktpolitische Instrumente - eingeteilt.

Nachfolgende Zusammenstellung von Maßnahmen (aus unterschiedlichen Literaturquellen) wurde entsprechend dieser Einteilung (vgl. Cerwenka et al. 2000) geordnet. Die darin aufgeführten Maßnahmen haben Beispielcharakter, der Anspruch auf Vollständigkeit wird nicht gestellt.

- Raumordungspolitische Maßnahmen

- Veränderung der Siedlungs- und Raumstruktur

- Abstimmung der Verkehrs- und Siedlungsplanung

- Verbesserung des Wohnumfeldes

- Verkehrsnachfragelenkung durch Flächennutzungsfestlegung

- Verkehrsinfrastrukturelle Maßnahmen

- Ausweitung des Angebotes auf der Schiene

- Vermeidung von (weiteren) (höherrangigen) Neu- und Ausbaumaßnahmen im Straßenbau

- Umgestaltung des Straßenraums

- Umgestaltung der Fahrbahnoberfläche

- Logistische Maßnahmen

- Maßnahmen zur besseren Auslastung der Fahrzeuge

- Maßnahmen im Bereich der Schienenlogistik, um Nachteile gegenüber dem Lkw abzubauen 
- Fahrzeugtechnische Maßnahmen

- Emissionsgrenzwerte für Fahrzeuge

- Senkung des Energiebedarfs bei Fahrzeugen

- Erhöhung des Wirkungsgrades

- Veränderung der Fahrzeugabmessungen und -gewichte

- Verkehrsordnungspolitische Maßnahmen

- Veränderung des Ordnungsrahmens

- Verbesserung und Durchsetzung der Kontrollen

- Vermeidung - Reduktion des Verkehrsaufkommens

- Fahrtenkontingentierung

- Beschränkung der Gewichte und Abmessungen von Fahrzeugen

- Emissionskontingentierung

- Fahrleistungskontingentierung

- zeitliche, sektorale und/oder lokale Fahrverbote

- Immissionsbedingte Fahrverbote bei Grenzüberschreitung

- zeitliche, sektorale und/oder lokale Tempolimite

- Punkteführerschein

- zentrales Führerscheinregister

- Preispolitische Maßnahmen

- Förderung von Ressourcen schonenderen Techniken

- Erleichterungen für den Einsatz neu entwickelter Techniken

- Öko-Bonus

- Abbau von Steuerprivilegien

- Verursacher gerechte Gestaltung der Haftpflichtversicherung

- Schwerverkehrsabgabe

- CO2-Abgabe

- Energie-Steuer/Abgabe (nach eingesetzter Energie)

- Emissionszertifikate (Emissionsrechte)

- Bevorzugte Investitionen im Infrastrukturausbau von umweltschonenden Verkehrsträgern

- Abbau von Wettbewerbsverzerrungen zw. den Verkehrsträgern (z.B. Wegekostenanlastung)

- Implementierung der externen Kosten in Mautgebührensätze 
- Implementierung der externen Kosten in Infrastrukturbenutzungsentgelte und Tarife im ÖPNV und Schienenverkehr

- Internationale Harmonisierung der Wettbewerbsbedingungen für Transporte

- Treibstoffzoll

- Autobahnvignette

- Motorfahrzeugsteuer

- Road-Pricing

- Kilometergebühr mit Grenzkostenabdeckung

- von technischen Standards abhängige Zulassungssteuern

- Stau abhängige Straßengebühr

- knappheitsabhängige Parkgebühr

- Kilometergebühr, differenziert nach Gewicht und Umweltbelastung

- Flächendeckende Kilometergebühr

- einheitliche Energiesteuer

- „Horizontal“ wirkende, begleitende Maßnahmen

- Maßnahmen zur Erhaltung der Sozialvorschriften

- Appelle und Anrufe

- Ausbildung und Erziehung

- Bewusstseinsbildung

- Bürgerbeteiligung

- Verkehrsforschung

- Nettoerträge aus Lenkungsabgaben refundieren

\subsubsection{Typologiebildung für umweltorientierte Verkehrslenkungsin- strumente im Güterverkehr}

Begriffsdefinition: Ein Instrumentarium ist die Gesamtheit zusammengehöriger Mittel/Werkzeuge, um einen Zweck/ein Ziel zu erreichen. Im Forschungsprojekt eingesetzt um verkehrspolitische Ziele zu erreichen.

Es wird nun versucht die umfassende Anzahl von Maßnahmen und ihrer vorerst noch recht feingliedrigen Einteilung ein übergeordnetes, einfaches System zu geben und die einzelnen Maßnahmenbündel in eine überblickbare und handhabbare Anzahl von Instrumentarien zusammenzufassen. Die Zuordnung erfolgt 
hier, wie bereits bei den Maßnahmenbündeln, nach den verwendeten Werkzeugen/Instrumenten.

Als Grundlage der Überlegungen für eine neue Systematik diente Hauger/Cerwenka et al. (2000) mit der Zusammenstellung der Wechselwirkung zwischen Raumnutzung und Raumüberwindung. Diese zeigt recht anschaulich die Beziehungen zwischen den einzelnen Umfeldern, in denen Verkehr als realisierte Raumüberwindung eingebettet ist.

Aus diesen Umfeldbereichen des Verkehrs in Verbindung mit den o.a. Maßnahmenbündeln wurde nun eine Einteilung erstellt, die sich auf die Gesellschaftsbereiche aufteilt und die als übergeordnete Systematik dienen soll. Die vier Instrumentarien lauten demnach (vgl. Tabelle 17):

- Sozio-Demografisches Instrumentarium

- Ökonomisches Instrumentarium

- Technologisches Instrumentarium

- Ökologisches Instrumentarium

Im Folgenden werden die einzelnen Instrumentarien näher erläutert.

\section{- Sozio-Demografisches Instrumentarium}

Dieses Instrumentarium sollte alle direkt den Menschen betreffenden Maßnahmen aufnehmen. Es sind dies die Umfeldbereiche Soziale Prozesse, Siedlungsstruktur und Bevölkerung.

\section{- Ökonomisches Instrumentarium}

Der Zusammenhang zwischen Wirtschaft und Verkehr ist komplex. Ein quantitativ und qualitativ gut ausgestattetes Verkehrssystem ist ganz entscheidend für die ökonomische Entwicklung. Daraus kann man im Umkehrschluss ein gewichtiges Instrumentarium in der Verkehrsnachfragebeeinflussung erkennen. Allerdings ist Wirtschaftswachstum nicht unbedingt an Verkehrswachstum gebunden (vlg. Cerwenka et al. 2000). Es werden damit also jene Maßnahmen erfasst, welche die Verkehrsnachfrage mit ökonomischen Mitteln zu verändern versuchen. 
Tabelle 17: Umweltorientierte Verkehrslenkungsinstrumente im Straßengüterverkehr nach Umfeldbereichen geordnet (Quelle: Cerwenka et al. 2000 stark erweitert und verändert)

\begin{tabular}{|c|c|c|c|}
\hline $\begin{array}{l}\text { GESELL- } \\
\text { SCHAFTSBE- } \\
\text { REICH }\end{array}$ & $\begin{array}{l}\text { POLITISCHER } \\
\text { RAHMEN }\end{array}$ & \multicolumn{2}{|c|}{$\begin{array}{l}\text { ZUGEHÖRIGE MASSNAHMEN } \\
\text { (AUSWAHL) }\end{array}$} \\
\hline \multirow[t]{2}{*}{$\begin{array}{l}\text { SOZIO- } \\
\text { DEMOGRAFIE }\end{array}$} & $\begin{array}{l}\text { ORDNUNGSPOLITISCHE } \\
\text { INSTRUMENTE }\end{array}$ & \multicolumn{2}{|c|}{$\begin{array}{l}\text { - Veränderung des Ordnungsrahmens } \\
\text { - Maßnahmen zur Einhaltung von Sozialvorschrif- } \\
\text { ten } \\
\text { - Verbesserung und Durchsetzung der Kontrollen } \\
\text { - Verkehrsvermeidung - Reduktion des Ver- } \\
\text { kehrsaufkommens: } \\
\text { - Veränderungen der Siedlungs- und Raumstruk- } \\
\text { tur } \\
\text { - Abstimmung der Verkehrs- und Siedlungspla- } \\
\text { nung } \\
\text { - Bürgerbeteiligung } \\
\text { - Bewusstseinsbildung } \\
\text { - Ausbildung und Erziehung } \\
\text { - Appelle und Anrufe }\end{array}$} \\
\hline & $\begin{array}{l}\text { MARKTPOLITISCHE } \\
\text { INSTRUMENTE }\end{array}$ & & \\
\hline \multirow[b]{3}{*}{ ÖKONOMIE } & $\begin{array}{l}\text { ORDNUNGSPOLITISCHE } \\
\text { INSTRUMENTE }\end{array}$ & \multicolumn{2}{|c|}{$\begin{array}{l}\text { - Fahrtenkontingentierung } \\
\text { - Förderung von ressourcenschonenderen Tech- } \\
\text { niken } \\
\text { - Erleichterungen für den Einsatz neu entwickelter } \\
\text { Techniken } \\
\text { - Förderung des öffentlichen Verkehrs } \\
\text { - Öko-Bonus } \\
\text { - Abbau von Steuerprivilegien } \\
\text { - Verursachergerechte Gestaltung der Haftpflicht- } \\
\text { versicherung } \\
\text { - Schwerverkehrsabgabe } \\
\text { - CO2-Abgabe }\end{array}$} \\
\hline & & $\begin{array}{l}\text { INFRASTRUK- } \\
\text { TURELLE } \\
\text { MASSNAHMEN }\end{array}$ & $\begin{array}{l}\text { Bevorzugte Investitionen im } \\
\text { Infrastrukturausbau von um- } \\
\text { weltschonenden Verkehrsträ- } \\
\text { gern }\end{array}$ \\
\hline & $\begin{array}{l}\text { MARKTPOLITISCHE } \\
\text { INSTRUMENTE }\end{array}$ & $\begin{array}{l}\text { PREISPOLITI- } \\
\text { SCHE MASS- } \\
\text { NAHMEN }\end{array}$ & $\begin{array}{l}\text { - Abbau von Wettbewerbsver- } \\
\text { zerrungen zw. Den Verkehrs- } \\
\text { trägern (z.B. Wegekostenan- } \\
\text { lastung) } \\
\text { - Implementierung der exter- } \\
\text { nen Kosten in Mautgebüh- } \\
\text { rensätzen } \\
\text { - Implementierung der exter- } \\
\text { nen Kosten auch in Infra- } \\
\text { strukturbenutzungsentgelte } \\
\text { und Tarife im ÖPNV und } \\
\text { Schienengüterverkehr } \\
\text { - Internationale Harmonisie- } \\
\text { rung der Wettbewerbsbedin- } \\
\text { gungen für Transporte (z.B. } \\
\text { einheitliche Besteuerung der } \\
\text { Lkw innerhalb Europas) } \\
\text { - Treibstoffzoll } \\
\text { - Autobahnvignette } \\
\text { - Motorfahrzeugsteuer } \\
\text { - Road-Pricing } \\
\end{array}$ \\
\hline
\end{tabular}


Fortsetzung Tabelle 17: Umweltorientierte Verkehrslenkungsinstrumente im Straßengüterverkehr nach Umfeldbereichen geordnet (Quelle: Cerwenka et al. 2000 stark erweitert und verändert)

\begin{tabular}{|c|c|c|c|}
\hline & & $\begin{array}{l}\text { LOGISTISCHE } \\
\text { MASSNAHMEN }\end{array}$ & $\begin{array}{l}\text { - Ausweitung des Angebotes } \\
\text { auf der Schiene } \\
\text { - Maßnahmen zur besseren } \\
\text { Auslastung der Fahrzeuge } \\
\text { (Transportbörsen, Einbezie- } \\
\text { hung des Werksverkehrs) }\end{array}$ \\
\hline \multirow{3}{*}{ TECHNOLOGIE } & $\begin{array}{l}\text { ORDNUNGSPOLITISCHE } \\
\text { INSTRUMENTE }\end{array}$ & \multicolumn{2}{|c|}{$\begin{array}{l}\text { - Emissionsgrenzwerte für Fahrzeuge } \\
\text { - Beschränkungen der Gewichte und Abmessun- } \\
\text { gen von Fahrzeugen } \\
\text { - Beschränkungen der Verbrauchswerte von } \\
\text { Fahrzeugen } \\
\text { - Verkehrsforschung }\end{array}$} \\
\hline & \multirow[b]{2}{*}{$\begin{array}{l}\text { MARKTPOLITISCHE } \\
\text { INSTRUMENTE }\end{array}$} & $\begin{array}{l}\text { INFRASTRUK- } \\
\text { TURELLE } \\
\text { MASSNAHMEN }\end{array}$ & $\begin{array}{l}\text { - Vermeidung von (weiteren) } \\
\text { (höherrangigen) Neu- und } \\
\text { Ausbaumaßnahmen im Stra- } \\
\text { ßenbau }\end{array}$ \\
\hline & & $\begin{array}{l}\text { LOGISTISCHE } \\
\text { MASSNAHMEN }\end{array}$ & $\begin{array}{l}\text { - Maßnahmen im Bereich der } \\
\text { Schienenlogistik, um Nachtei- } \\
\text { le gegenüber dem Lkw abzu- } \\
\text { bauen (Verwendung moder- } \\
\text { ner Techniken des unbeglei- } \\
\text { teten kombinierten Verkehrs) }\end{array}$ \\
\hline \multirow[t]{2}{*}{ ÖKOLOGIE } & $\begin{array}{l}\text { ORDNUNGSPOLITISCHE } \\
\text { INSTRUMENTE }\end{array}$ & \multicolumn{2}{|c|}{$\begin{array}{l}\text { - Emissionskontingentierung } \\
\text { - Fahrtenkontingentierung } \\
\text { - zeitliche, sektorale und/oder lokale Fahrverbote } \\
\text { - Immissionsbedingte Fahrverbote bei Grenzüber- } \\
\text { schreitung } \\
\text { - zeitliche, sektorale und/oder lokale Tempolimite } \\
\text { - Verbesserung des Wohnumfeldes } \\
\text { - Emissionszertifikate }\end{array}$} \\
\hline & $\begin{array}{l}\text { MARKTPOLITISCHE } \\
\text { INSTRUMENTE }\end{array}$ & & \\
\hline
\end{tabular}

\section{- Technologisches Instrumentarium}

Im technologischen Instrumentarium sind all jene Maßnahmen zusammengefasst, welche ihre Aufgaben mit technischen Mitteln und Veränderungen realisieren. Der Begriff Technisches Instrumentarium stellt einen Überbegriff dar.

\section{- Ökologisches Instrumentarium}

Erst in den letzten Jahren wurde der Umweltaspekt ein immer stärker werdender Einflussparameter auf das Verkehrsgeschehen. Sowohl in der Planung und Errichtung von Verkehrsinfrastruktur als auch in der technischen Weiterentwicklung von Rollmaterial dominieren heute, gestützt und gefördert von Politik und Recht, Aspekte eines schonenden Umganges mit begrenzt verfügbaren Umweltressourcen. Daraus lässt sich nun wieder ableiten, welche Maßnahmen gesetzt werden müssten, um im Sinne der Ökologie in das Verkehrsgeschehen einzugreifen. Beispielhaft dafür können genannt werden: soziales Lernen, Rechtsnormen, diverse Regulative, und vor allem Maßnahmen die direkten Einfluss auf die schädlichen Auswirkungen des Straßenverkehrs haben. 


\subsection{Normativer Rahmen zu Emissionen}

Tabelle 18: Überblick über die wesentlichen und für das Forschungsprojekt relevanten normativen Festlegungen von Grenzwerten von Luftschadstoffen

\begin{tabular}{|c|c|c|c|}
\hline GESETZGEBER & NR. & BEZEICHNUNG & ANMERKUNG \\
\hline \multirow[t]{4}{*}{ EU Rat } & $\begin{array}{l}\text { Rahmenrichtlinie Luftqua- } \\
\text { lität 96/62/EG vom } 27 \text {. } \\
\text { September } 1996\end{array}$ & $\begin{array}{l}\text { RL 96/62/EG des Rates } \\
\text { über die Beurteilung und } \\
\text { die Kontrolle der Luftqua- } \\
\text { lität }\end{array}$ & $\begin{array}{l}\text { Als explizit zu prüfen- } \\
\text { de Luftschadstoffe } \\
\text { werden SO2, NO2, } \\
\text { Feinpartikel, Schweb- } \\
\text { staub, Blei und Ozon } \\
\text { angeführt, die in der } \\
\text { Anfangsphase zu } \\
\text { prüfen sind. }\end{array}$ \\
\hline & $\begin{array}{l}\text { Richtlinie 1999/30/EG } \\
\text { vom 22. April 1999 }\end{array}$ & $\begin{array}{l}\text { RL 1999/30/EG über } \\
\text { Grenzwerte für Schwefel- } \\
\text { dioxid, Stickstoffdioxid } \\
\text { und Stickstoffoxide, Parti- } \\
\text { kel und Blei in der Luft }\end{array}$ & $\begin{array}{l}\text { Grenzwerte für SO2 } \\
\text { und Partikel gelten ab } \\
2005 \text {, für NOx ab } \\
2010\end{array}$ \\
\hline & $\begin{array}{l}\text { Richtlinie } 2000 / 69 / E G \\
\text { vom } 16 . \text { November } 2000\end{array}$ & $\begin{array}{l}\text { RL 200/69/EG über } \\
\text { Grenzwerte für Benzol } \\
\text { und Kohlenmonoxid in der } \\
\text { Luft }\end{array}$ & $\begin{array}{l}\text { Grenzwert für Benzol } \\
\text { gilt ab 2010, für Koh- } \\
\text { lenmonoxid ab } 2005\end{array}$ \\
\hline & $\begin{array}{l}\text { Richtlinie } 2002 / 2 / \text { EG vom } \\
\text { 12. Februar } 2002\end{array}$ & $\begin{array}{l}\text { RL 2002/3/EG über den } \\
\text { Ozongehalt der Luft }\end{array}$ & $\begin{array}{l}\text { Die RL enthält Ziel- } \\
\text { werte, die } 2010 \text { er- } \\
\text { reicht werden sollten }\end{array}$ \\
\hline \multirow[t]{2}{*}{$\begin{array}{l}\text { Republik Öster- } \\
\text { reich }\end{array}$} & $\begin{array}{l}\text { BGBI. I Nr. 115/1997 in } \\
\text { der Fassung BGBI. I Nr. } \\
34 / 2003\end{array}$ & $\begin{array}{l}\text { Bundesgesetz zum } \\
\text { Schutz vor Immissionen } \\
\text { durch Luftschadstoffe, mit } \\
\text { dem die Gewerbeordnung } \\
\text { 1994, das Luftreinhalte- } \\
\text { gesetz für Kesselanlagen, } \\
\text { das Berggesetz 1975, das } \\
\text { Abfallwirtschaftsgesetz } \\
\text { und das Ozongesetz } \\
\text { geändert werden (Immis- } \\
\text { sionsschutzgesetz -Luft, } \\
\text { IG-L) }\end{array}$ & $\begin{array}{l}\text { Die Ausführungen zu } \\
\text { verkehrsbedingten } \\
\text { Emissionen sind auf } \\
\text { einen Paragrafen } \\
\text { beschränkt (\$22). }\end{array}$ \\
\hline & $\begin{array}{l}\text { BGBI. Nr. 210/1992 in der } \\
\text { Fassung BGBI. I Nr. } \\
34 / 2003\end{array}$ & $\begin{array}{l}\text { Bundesgesetz über Maß- } \\
\text { nahmen zur Abwehr der } \\
\text { Ozonbelastung und die } \\
\text { Information der Bevölke- } \\
\text { rung über hohe Ozonbe- } \\
\text { lastungen, mit dem das } \\
\text { Smogalarmgesetz, BGBI. } \\
\text { Nr. 38/1989, geändert } \\
\text { wird (Ozongesetz) }\end{array}$ & $\begin{array}{l}\text { Es ist eine Reduktion } \\
\text { der NOx-Emissionen } \\
\text { um } 40 \% \text { bis } 1996, \text { um } \\
60 \% \text { bis } 2001 \text { und um } \\
70 \% \text { bis } 2006 \text {, jeweils } \\
\text { bezogen auf die } \\
\text { Emissionen des } \\
\text { Jahres } 1985 \text { ( } 234.000 \\
\text { Tonnen) vorgesehen. } \\
\text { Der dann erreichte } \\
\text { Level von 103.000 } \\
\text { Tonnen (vgl. BGBI. I } \\
\text { Nr. 34/2003) liegt } \\
\text { unter dem Göteborg- } \\
\text { ziel* }\end{array}$ \\
\hline
\end{tabular}




\begin{tabular}{|c|c|c|c|}
\hline $\begin{array}{l}\text { Republik Öster- } \\
\text { reich }\end{array}$ & BGBI. II Nr. 298/2001 & $\begin{array}{l}\text { Verordnung des Bundes- } \\
\text { ministers für Land- und } \\
\text { Forstwirtschaft, Umwelt } \\
\text { und Wasserwirtschaft } \\
\text { über Immissionsgrenz- } \\
\text { werte und Immissions- } \\
\text { zielwerte zum Schutz der } \\
\text { Ökosysteme und der } \\
\text { Vegetation }\end{array}$ & $\begin{array}{l}\text { Es sind Grenz- und } \\
\text { Zielwerte für die } \\
\text { Schadstoffe SO2 und } \\
\text { NOx angegeben. }\end{array}$ \\
\hline & BGBI. I Nr. 34/2003 & $\begin{array}{l}\text { Bundesgesetz, mit dem } \\
\text { das Bundesgesetz über } \\
\text { nationale Emissions- } \\
\text { höchstgrenzen für be- } \\
\text { stimmte Luftschadstoffe } \\
\text { (Emissionshöchstmen- } \\
\text { gengesetz-Luft, EG-Luft) } \\
\text { erlassen sowie das O- } \\
\text { zongesetz und das IG- } \\
\text { Luft geändert wird. }\end{array}$ & $\begin{array}{l}\text { Es sind die nationalen } \\
\text { Höchstmengen der } \\
\text { Emissionen für SO2, } \\
\text { NOx, VOC (flüchtige } \\
\text { organische Verbin- } \\
\text { dungen) und Ammo- } \\
\text { niak (NH3) festgelegt. } \\
\text { SO2: } 39.000 \text { to, NOx } \\
103.000 \text { to, VOC } \\
159.000 \text { to, NH3 } \\
66.000 \text { to }\end{array}$ \\
\hline
\end{tabular}

* Das Göteborgziel legt für 2010 einen Zielwert von 107.000 Tonnen/Jahr fest

\subsection{Verwendete und entwickelte Methoden}

Bezüglich der zum Einsatz kommenden Untersuchungsmethoden unterscheiden sich die beiden Untersuchungsphasen deutlich:

- In Untersuchungsphase 1, in der die Aufbereitung der Problemsituation erfolgen muss, dominiert das analytische Instrumentarium (Dokumentenanalyse, Beobachtung, Berücksichtigung und Einarbeitung der problemrelevanten gesellschaftlichen Entwicklungen, Kompatibilitätsprüfungen, Heranführung an den aktuellen Stand des Geschehens).

- In Untersuchungsphase 2, in der das aus Umweltschutzgründen für notwendig erachtete verkehrspolitische Lenkungsinstrument entwickelt werden muss, werden sowohl analytische Methoden, rechnergestützte Simulationen als auch Elemente aus Kreativitäts -und Szenarientechniken zur Anwendung kommen.

Beiden Untersuchungsphasen gemeinsam sind die Tatsache, dass hier ein in der realen Welt vorliegendes komplexes Gestaltungsproblem mit Hilfe der Wissenschaft einer Lösung zugeführt werden muss. 


\section{Problem $>$ Modellierung $>$ Problemlösung}

(analog MÜLLER - MERBACH)

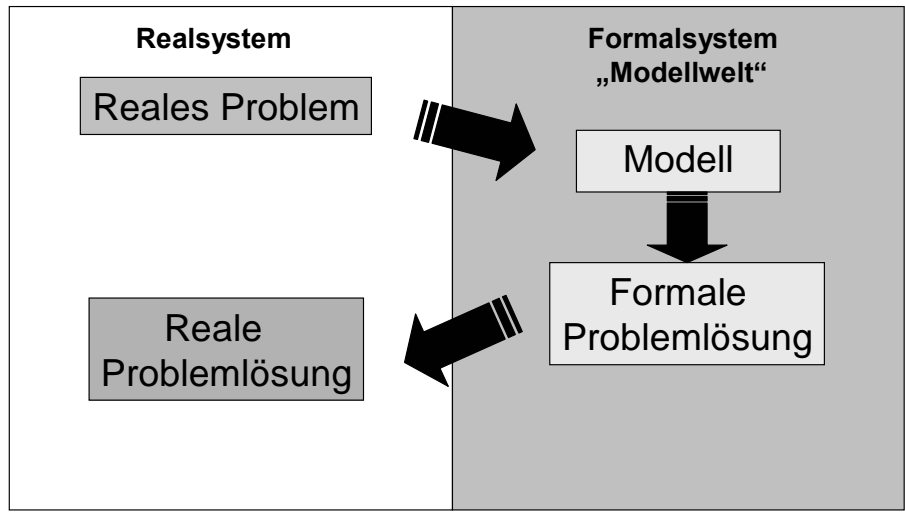

Abbildung 28: Modellierung als Zwischenschritt bei der Suche nach einer realen Problemlösung (Vgl. Müller-Merbach, Heiner, Operations Research, 2. Aufl., München 1971, S. 14).

Die Modellierung der konkret zu lösenden Gestaltungsaufgabe ist in solchen Fällen unerlässlich. Das Modell als vereinfachte Abbildung der real vorhandenen Entscheidungssituation hat die Funktion eines Gestaltungsinstrumentes zu übernehmen. MÜLLER-MERBACH veranschaulicht treffend (vgl. Abbildung 28), dass in vielen Fällen die Realsituation zwar nach einer realen Lösung verlangt, diese reale Lösung aber nicht auf direktem Wege ermittelt werden kann; der Weg zur realen Problemlösung führt über das Formalsystem, wo mit Hilfe eines Modells eine formale Problemlösung gefunden wird, die in der Folge die Basis für die reale Problemlösung bildet. Dieser Weg muss auch bei der vorliegenden Aufgabenstellung beschritten werden:

- Das reale Problem besteht in diesem Falle darin, dass für sensible Gebiete, wie z.B. den alpinen Raum, in punkto Schadstoffbelastung eine spezielle Obergrenze fixiert werden muss, damit die Regenerationsfähigkeit der Natur erhalten bleibt. Angesichts der Komplexität des Systems, in das mit einer solchen Regulierungsmaßnahme eingegriffen werden muss, ist davon auszugehen, dass die reale Problemlösung nicht ein Schnellschuss sein kann, sondern unter sorgfältiger Beachtung der multikausalen Wirkungsverflechtung und der vorhandenen Systemdynamik entwickelt werden muss. Dies bedeutet, dass der Weg zur Problemlösung über die in Abbildung 28 genannten Schritte erfolgen muss.

- Die Modellbildung spielt sich im Formalsystem ab. Das zu entwickelnde Modell muss die reale Problemsituation abbilden, die in diesem Falle eine Entscheidungssituation ist: Die Regulierungsmaßnahme wird beim Errei- 
chen einer bestimmten Schadstoffbelastung notwendig; aber sie soll in Kenntnis der von ihr zu erwartenden Auswirkungen auf die Systembereiche Wirtschaft, Gesellschaft und Umwelt erfolgen.

- Das für die vorhandene Entscheidungssituation zu entwickelnde Modell wird es ermöglichen, eine formale Problemlösung zu finden. Diese formale Problemlösung wird darin bestehen, dass das Modell die Spezifikation jener Regulierungsmaßnahme gestattet, die im Falle ihrer Umsetzung die gesamtgesellschaftlich optimale Entscheidungsvariante wäre.

- Die reale Problemlösung als das eigentliche Ziel der wissenschaftlichen Entscheidungsvorbereitung spielt sich im Realsystem ab, und zwar dadurch, dass die gefundene formale Problemlösung auf das Realsystem übertragen wird.

Nachfolgend sind in Tabelle 19 bis Tabelle 21, getrennt nach den einzelnen Fachbereichen, die möglichen Untersuchungsmethoden aufgelistet. Die Methoden sind nach Bedarf im Einzelnen kurz erläutert. Zusätzlich wird für den entsprechenden Fachbereich dargestellt, welche aktuellen Rahmenbedingungen und Einflussfaktoren im gegenständlichen Forschungsprojekt Berücksichtigung finden.

Tabelle 19: Zusammenstellung der angewandten Untersuchungsmethoden für den Fachbereich Verkehrsplanung

\begin{tabular}{|c|c|c|}
\hline 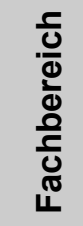 & VERKEHRSPLANUNG & 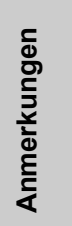 \\
\hline \multicolumn{3}{|c|}{ METHODEN: } \\
\hline & $\begin{array}{l}\text { Vergleichen und Kontrastieren für das Gegenüberstellen von Sachverhalten, } \\
\text { Ähnlichkeiten und Unterschieden }\end{array}$ & \\
\hline & $\begin{array}{l}\text { Analysieren der vorhandenen Lenkungsinstrumente in abstrakte Bezüge und } \\
\text { Eigenschaften }\end{array}$ & \\
\hline & \multirow{2}{*}{$\begin{array}{l}\text { Bewerten von Sachverhalten und Lösungsansätzen: } \\
\text { - Wirkungsanalyse } \\
\text { - Nutzwertanalyse }\end{array}$} & \\
\hline & & \\
\hline & Szenarioanalyse und Szenariotechnik & \\
\hline & Sensitivitätsanalyse zur Überprüfung der Stabilität der Ergebnisse & \\
\hline \multicolumn{3}{|c|}{$\begin{array}{l}\text { FEATURE: Problemrelevante Gesellschafts-, wirtschafts- und umweltpolitische Entwick- } \\
\text { lungen, die Berücksichtigung finden: }\end{array}$} \\
\hline & \multicolumn{2}{|l|}{$\begin{array}{l}\text { Die Emissionen durch den Betrieb von einzelnen Verkehrsträgern eignen sich als Basis für ein Len- } \\
\text { kungsinstrument für den Güterverkehr durch ökologisch sensible Gebiete. Ein Emissionsbegren- } \\
\text { zungsmodell kann einen Beitrag zur Erfüllung der Kyoto-Ziele und anderer normativer Festlegungen } \\
\text { liefern. }\end{array}$} \\
\hline
\end{tabular}


Tabelle 20: Zusammenstellung der angewandten und diskutierten Untersuchungsmethoden für den Fachbereich Verkehrsökologie

\begin{tabular}{|c|c|c|}
\hline $\begin{array}{l}\frac{1}{0} \\
\frac{0}{0} \\
\frac{0}{0} \\
\frac{0}{0} \\
\frac{1}{0} \\
\text { I }\end{array}$ & VERKEHRSÖKOLOGIE & 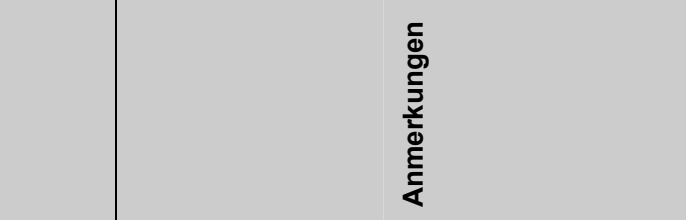 \\
\hline \multicolumn{3}{|c|}{ METHODEN: } \\
\hline & \multirow[b]{2}{*}{$\begin{array}{l}\text { Verträglichkeitsanalysen: } \\
\text { - } \quad \text { Modell der autonomen und re- } \\
\text { - } \text { lativen Standards } \\
\text { - } \quad \text { Kodir-Verfahren } \\
\text { - } \quad \text { Berlinensatorischer Ansatz } \\
\end{array}$} & \\
\hline & & $\begin{array}{l}\text { Methoden aus der kommunalen Verkehrsplanung, } \\
\text { deren Grundüberlegungen und Ausgangspunkt, näm- } \\
\text { lich der Nutzungskonflikt zwischen Verkehr und das } \\
\text { inn umgebende Gebiet ist. Dieser Ansatz ist zur Be- } \\
\text { stimmung der Verträglichkeit von Straßengüterverkehr } \\
\text { in ökologisch sensiblen Gebieten als Basis verwend- } \\
\text { bar. Eine wissenschaftliche Weiterentwicklung ist Teil } \\
\text { der gegenständlichen Forschungsaufgabe. }\end{array}$ \\
\hline & Ökologische Risikoanalyse & $\begin{array}{l}\text { Diese Methode ist der „Versuch einer planerischen } \\
\text { Operationalisierung des Verursacher-Auswirkung- } \\
\text { Betroffener-Zusammenhangs, d.h. eine Form der } \\
\text { Wirkungsanalyse im Mensch-Umwelt-System“ (Bach- } \\
\text { fischer 1978). Eine formale Beurteilung erfolgt durch } \\
\text { Bildung der drei „Aggregatsgrößen“ Intensität der } \\
\text { Beeinträchtigung, Empfindlichkeit gegenüber Beein- } \\
\text { trächtigungen und dem Risiko der Beeinträchtigung. }\end{array}$ \\
\hline & Methode der ökologischen Knappheit & $\begin{array}{l}\text { Eine „Distance-to-Target“ Methode, die versucht mit } \\
\text { standardisierten, allgemein anwendbaren Gewichtun- } \\
\text { gen zu arbeiten. Diese Methode strebt eine Optimie- } \\
\text { rung im Rahmen der umweltpolitischen Ziele an. } \\
\text { Dabei wird vorausgesetzt, dass die Emissionsvor- } \\
\text { schriften eingehalten sind. }\end{array}$ \\
\hline & $\begin{array}{l}\text { „Dynamischer ökologischer“ Fußab- } \\
\text { druck }\end{array}$ & $\begin{array}{l}\text { Der "dynamische ökologische“ Fußabdruck ist ein } \\
\text { Maß zur Veranschaulichung der ökologischen Ver- } \\
\text { träglichkeit der NOx-Emissionen des Straßengüter- } \\
\text { verkehrs in einem definierten Gebietsabschnitt. Er } \\
\text { stellt ein fiktives Flächenäquivalent dar, welches die } \\
\text { notwendige Fläche zur Kompensation der durch die } \\
\text { betrieblich verursachten NOx-Emissionen auftreten- } \\
\text { den Schadenswirkungen des Straßengüterverkehrs } \\
\text { repräsentiert, und zwar in Abhängigkeit: } \\
\text { der spezifischen normativen Bestimmungen, der } \\
\text { lokalen Eigenschaften des Gebietsabschnittes und } \\
\text { der Infrastruktur und der zeitlichen Verteilung und der } \\
\text { technischen Zusammensetzung des Straßengüterver- } \\
\text { kehrs (Frewein 2005, S. 188) }\end{array}$ \\
\hline \multicolumn{3}{|c|}{$\begin{array}{l}\text { FEATURE: Problemrelevante Gesellschafts-, wirtschafts- und umweltpolitische Entwick- } \\
\text { lungen, die Berücksichtigung finden: }\end{array}$} \\
\hline & \multicolumn{2}{|c|}{$\begin{array}{l}\text { Ökologisch sensible Gebiete im Kontext mit dem Straßengüterverkehr werden beim Prinzip der Be- } \\
\text { aufschlagung (Wegekosten) in ökosensiblen Zonen bereits im derzeitigen österreichischen Regie- } \\
\text { rungsprogramm (Regierungsprogramm der österreichischen Bundesregierung für die XXII Gesetzge- } \\
\text { bungsperiode, 2003-2006) berücksichtigt. Für diesen Ansatz von nachhaltigen Überlegungen ist also } \\
\text { bereits eine gesellschaftliche Anerkennung da. Ob, wann und wie diese Bekenntnisse „Umsetzungs- } \\
\text { reife“ erlangen, ist schwer abzusehen, denn gerade für eine Identifizierung von ökologisch sensiblen } \\
\text { Gebieten ist die Abwägung der regionalen Tragfähigkeit und Sensibilität und der Belastung des Gebie- } \\
\text { tes entscheidend. Ein dafür geeignetes Ziele-Kriterien-Indikatoren System, als Diskussionsvorschlag } \\
\text { zu entwickeln unter dem Prinzip der Nachhaltigkeit, ist auf Basis der angeführten Methoden möglich. }\end{array}$} \\
\hline
\end{tabular}


Tabelle 21: Zusammenstellung der angewandten und diskutierten Untersuchungsmethoden für den Fachbereich Verkehrsrecht und rechtliche Grundlagen

\begin{tabular}{|c|c|c|}
\hline 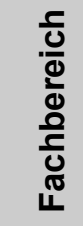 & RECHTLICHER TEIL: & 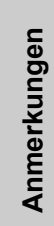 \\
\hline \multicolumn{3}{|c|}{ METHODEN: } \\
\hline & $\begin{array}{l}\text { Rechts-Dogmatik (Problemzentrierte Analyse des geltenden Rechts und der } \\
\text { Probleme der Rechtsprechung): } \\
\text { - Der Rechtsschutz des Nachbarn gegenüber Emissionen aus dem Be- } \\
\text { trieb von Straßen } \\
\text { - Darstellung der Probleme der Emissionsvermeidung an Autobahnen als } \\
\text { Rechtsproblem }\end{array}$ & \\
\hline & $\begin{array}{l}\text { Rechtspolitik: } \\
\text { - Entwurf der Grundzüge eines österreichischen Anlagenrechts für Stra- } \\
\text { Benverkehrsanlagen nach den Grundsätzen des europäischen Umwelt- } \\
\text { Anlagenrechts (IPPC-RL) als systemgerechte Lösung im österreichi- } \\
\text { schen und europäischen Umweltrecht } \\
\text { - Untersuchung der europarechtlichen Rahmenbedingungen, insbeson- } \\
\text { dere der Kompetenzlage der Mitgliedsstaaten und der Europäischen } \\
\text { Gemeinschaft zur Entwicklung einer Umweltpolitik mittels Straßen- } \\
\text { Verkehrsanlagenrecht. }\end{array}$ & \\
\hline & $\begin{array}{l}\text { Rechtstatsachenforschung: } \\
\text { - Mitentwicklung von Kriterien für Steuerungsinstrumente zur lokalen Be- } \\
\text { grenzung von Verkehrsemissionen in Zusammenarbeit mit den im Pro- } \\
\text { jekt vertretenen Wissenschaften } \\
\text { - Mitentwicklung von Kriterien für die „ökologische Kapazität von Stra- } \\
\text { Benabschnitten“ (insbes. in sensiblen Gebieten) zur Verkehrssteue- } \\
\text { rung: wie viele Fahrzeuge welcher Emissionsklasse können zu einer } \\
\text { bestimmten Zeit einen bestimmten Abschnitt der Autobahn unter wel- } \\
\text { chen Fahrbedingungen benützen, damit die jeweilig gültigen Grenzwer- } \\
\text { te (Lärm, Schadstoffe) eingehalten werden können? } \\
\text { Untersuchung der rechtlichen Grundlagen für die Installation eines an- } \\
\text { lagenbezogenen Durchfahrtsrechte/Emissionsrechte - Handels. }\end{array}$ & \\
\hline
\end{tabular}

\subsection{Neue Eurovignetten Richtlinie 2006/38/EG: Kurze Chronologie}

Die EK hat diesen als wichtigen Bestandteil der zukünftigen gemeinschaftlichen Verkehrspolitik vorgelegt. Der Vorschlag möchte vor allem die Wettbewerbsbedingungen harmonisieren, insbesondere innerhalb der verschiedenen Verkehrsträger. Dabei wird auf die Kostenstrukturen eingegangen, die nach verschiedenen Parametern differenziert werden. Weiters möchte man durch den Vollkostenansatz die Kosten der Infrastrukturnetze decken und unter Einbeziehung des Binnenmarktaspektes die bessere Ausnutzung der Infrastruktur erreichen.

Im Verlauf der folgenden Diskussionen des Vorschlages in den Ratsarbeitsgruppen hatten sich einige wichtige Fragestellungen zu Hauptdiskussionspunkten herauskristallisiert: 
der Anwendungsbereich der Richtlinie, wie Fragen des betroffenen Netzes und der betroffenen Fahrzeuggruppen,

die Auswirkungen der Richtlinie auf bestehende nationale Gebührensysteme, die anrechenbaren Kosten,

die Einbeziehung anderer als infrastrukturbedingter Kosten in das Gebührensystem,

- Einbindung von externen Kosten des Verkehrs,

- der Grundsatz der Gebührenerhöhung in sensiblen Gebieten mit der Möglichkeit zur Anschubfinanzierung alternativer Infrastrukturen im selben Korridor,

- die Variationsmöglichkeit der Gebühren zur Stauvermeidung und nach Umweltkriterien,

- die Verwendung der Mauteinnahmen

- und andere Punkte.

Diskussion ergab sich anfangs auch zur Frage der Rechtsgrundlage, ob Artikel 71 des EU-Vertrages (qualifizierte Mehrheit) ausreicht, oder auf Grund des im Entwurf enthaltenen Bezuges auch zu steuerlichen Aspekten Artikel 93 (Einstimmigkeit) anzuwenden wäre.

Der Juristische Dienst der Kommission ist dazu zu der klaren Auffassung gelangt, dass im Änderungsvorschlag die Aspekte der Gebühren und Kostenfragen gegenüber den geringfügigen steuerlichen Gesichtspunkten eindeutig hoch überwiegen und daher Artikel 71 (qualifizierte Mehrheit) anzuwenden wäre.

Der Europäische Rat hatte in seiner Sitzung am 12. Dezember 2003 den Rat Verkehr aufgefordert, möglichst bis zum März 2004 zu einem einheitlichen Standpunkt zu finden.

Der Rat (Verkehr, Telekommunikation und Energie) ist am 9. März 2004 in Brüssel bei diesem Dossier aber zu keiner Einigung gekommen, wenngleich man dieser bis auf die Frage der Zweckbindung der Gebühreneinnahmen nahe war.

Das Europäische Parlament hat seine Stellungnahme in erster Lesung auf seiner Plenartagung vom 19. bis 22. April 2004 angenommen.

Zur Ratssitzung am 11. Juni 2004 in Luxemburg hatte der Irische Ratsvorsitz einen etwas abgeänderten Kompromissvorschlag vorgelegt. In der Diskussion, an der erstmals auch die neuen Mitgliedstaaten stimmberechtigt teilnahmen, wurden aber erneut viele Fragen aufgeworfen, sogar solche, die bereits als mehr oder weniger gelöst galten, sodass erneut keine Einigung im Rat zustande kam.

Unter Niederländischem Ratsvorsitz war erneut eine Abstimmung über die Richtlinienänderung auf die Tagesordnung der Ratssitzung vom 7. Oktober 2004 in Luxemburg gesetzt worden. Der vorgelegte gegenüber Juni 2004 etwas modifizierte Vorschlag fand aber mehrheitliche wieder keine Zustimmung der Mitgliedstaaten. 


\subsubsection{Einbeziehung der externen Kosten des Verkehrs}

Ausgangspunkt für die Diskussion ist der „Entwurf einer Empfehlung für die zweite Lesung im Europäischen Parlament “50:

Im Änderungsantrag 2 wird ausdrücklich betont: „Der Europäische Rat hat auf seiner Tagung vom 15. und 16. Juni 2001 in Göteborg in Ziffer 29 seiner Schlussfolgerungen festgehalten, dass eine nachhaltige Verkehrspolitik dem Anstieg des Verkehrsaufkommens und der Verkehrsüberlastung, des Lärms und der Umweltverschmutzung entgegenwirken und die Verwendung umweltfreundlicher Verkehrsmittel sowie die vollständige Internalisierung der sozialen und der Umweltkosten fördern sollte."

Abgesehen von der nicht sehr präzisen Definition der Umweltkosten wird nachdrücklich auf die Förderung der vollständigen Internalisierung der sozialen und der Umweltkosten hingewiesen.

Änderungsantrag 6: „(14a) Um die künftige Anwendung des Verursacherprinzips für alle Verkehrsträger zu gewährleisten sollten einheitliche auf wissenschaftlich anerkannte Daten basierende Prinzipien für die Berechnung entwickelt werden, die den Weg für die Internalisierung externer Kosten frei machen."

Ein entscheidender Punkt für der Einbeziehung der externen Kosten des Verkehrs ist dabei der Standpunkt des Europäischen Parlaments, dass es zur Zeit nicht möglich sei, diese Kosten einigermaßen einheitlich und korrekt zu erfassen und berechnen, was immer wieder ein Grund zur Ablehnung der Einbeziehung der externen Kosten führte.

Dabei muss man allerdings verstehen, um welche Größenordnungen es dabei geht:

Während auf dem österreichischen Straßennetz (Autobahnen, Schnellstraßen, verländerte Bundesstraßen B, Landes- und Gemeindestraßen) die Infrastrukturkosten ca. 5 Mrd. Euro pro Jahr ausmachen ${ }^{51}$, während sich (bei moderater Rechnung) die dazu gehörenden externen Kosten auf das Doppelte, nämlich $10 \mathrm{Mrd}$. Euro pro Jahr belaufen ${ }^{52}$ !

\footnotetext{
${ }^{50}$ „Entwurf einer Empfehlung für die zweite Lesung im Europäischen Parlament“ betreffend den Gemeinsamen Standpunkt des Rates im Hinblick auf den Erlass der Richtlinie des Europäischen Parlaments und des Rates zur Änderung der Richtlinie 1999/62/EG über die Erhebung von Gebühren für die Benutzung bestimmter Verkehrswege durch schwere Nutzfahrzeuge; Berichterstatterin: Corien Wortmann-Kool (9856/2005 - C6-0000/2005 - 2003/0175(COD))

${ }^{51}$ HERRY M. (2005): Österreichische Wegekostenrechnung 2000 - Was ist hier so teuer. In: Schriftenreihe der Institute „Eisenbahnwesen und Verkehrswirtschaft“ und „Straßen- und Verkehrswesen“ der TU Graz, Heft 28

${ }^{52}$ HERRY M., SEDLACEK N. (2003): Österreichische Wegekostenrechnung für die Straße 2000. Bundesministerium für Verkehr, Innovation und Technologie, Straßenforschung, Heft 528, Wien
} 
Dabei geht es weniger darum, all diese Kosten sofort zur Gänze richtlinienmäßig einzufordern, sondern eine schrittweise, aber verpflichtende Einbindung anzugehen.

In den Änderungsanträgen vom 23.9.2005 wird nun vorgeschlagen:

Änderungsantrag 51: „Der Wert externer Kosten muss sich aus auf europäischem Niveau angenommenen objektiv quantifizierten Berechnungsmethoden ergeben. So lange eine solche Methode noch nicht genehmigt wurde, können die Mitgliedsstaaten höchstens 60\% der Infrastrukturkosten für die Berechnung externer Kosten ansetzen."

Dieser Vorschlag geht auf die Initiative „Open Letter of European Scientists in the Field of Transport and Economy Research To The Members of the European Parliament, The Transport Ministers of the EU Member States The Commissioner for Transport, vice-president of the European Commission M. Jacques Barrot concerning Internalisation of external costs in the scope of the new "Directive on Charging of Heavy Goods Vehicles "(1999/62/EG)"53 zurück.

Im Änderungsantrag 16 schließlich wird empfohlen:

ARTIKEL 1 NUMMER 8: „Die Kommission legt bis spätestens 3 Jahre nach der [Umsetzungsfrist dieser Richtlinie] ein allgemein gültiges, transparentes und nachvollziehbares Modell zur Monetarisierung aller externen Umwelt-, Stau- und Gesundheitskosten vor, welches künftigen Berechnungen von Infrastrukturgebühren zugrunde gelegt wird. Dieses Modell wird durch eine Analyse der Auswirkungen auf die Internalisierung externer Kosten für alle Verkehrsträger begleitet.“

\footnotetext{
53 With great interest, we follow the current revision of the "Directive on Charging of Heavy Goods Vehicles" (1999/62/EG). According to our information some members of the European Parliament and the Council of Ministers argue that an accepted method for the internalisation of external costs does not exist. Therefore, the European Parliament and the Council of Ministers conclude that the concrete internalisation of external costs cannot be does not exist. Therefore,

We, European scientists and experts in this field of research ask you to accept the following comments:

1. There is a wide agreement among scientists that external costs should be internalised in order to make the economy more efficient.

2. An enormous amount of research has taken place into the measurement of external costs in recent years, as a result of which a broad consensus on the appropriate approach to measurement now exists. It is not true that a uniform calculation method for the charge of external costs is impossible. Scientifically, a reliable and uniform method for charging external costs can be agreed on within a short time and we regard this to be indispensable. 3 . There is a broad basic consensus among the scientists in Europe concerning the minimum level of external costs. The most cautious studies estimate total external costs for air pollution, noise and accidents at a level of $60 \%$ of the infrastructure costs. Only the upper limits need still more examination.

4. There is still a need for further research and discussions, and it is certain that agreement will not be absolutely unanimous, which is not even the case for commercial accounting conventions. However, this does not justify any further delay for taking concrete measures. It is better to internalise external costs based on a non perfect model than to neglect them completely. Any further delay has negative impacts on the economy as a whole. Continuous discussions and improvements characterise any serious research and policy applications.

5. Reflecting these results, we recommend the immediate implementation of the polluter pays principle into the framework of the Directive. This could be realised by using a phase-in of charging for external costs, e.g. an annual increase of the fee, e.g. of $5-8 \%$ p.a., over a period of $5-10$ years. This period should be used for continuing research and adaptation of the common method for the internalisation of external costs and examination of its effects.

6 . Besides, we recommend a convincing communication strategy including all stakeholders, in order to increase the acceptance of the taken measures. An important content should be estimates of the benefits of such a pricing policy in terms of reduced congestion and environmental improveures. An important content should be estimates of the benefits of such a pricing policy in terms of reduced congestion and environmental improve-
ment.

7. The sciences have made their contribution to this overdue process; a serious political will to introduce the polluter pays principle is now needed. Summary

External costs of the road transport sector can and should be internalised within a short time. It is scientifically possible and long overdue to decide on a method for a step wise implementation of charging for external costs and to introduce this into the current revision of the "Directive on charging of heavy vehicles" now.

If the inclusion of external costs should be adopted we will be happy to contribute to the development of a uniform method for charging external costs.
} 
Dazu ist festzustellen, dass es bereits eine Reihe von EU-Projekten zu diesem Thema gibt, wie z.B. UNITE. Solche Unternehmungen haben jedoch gezeigt, dass es zumindest kurz- und mittelfristig - nicht möglich sein wird, dieses Ziel zu erreichen.

Im Änderungsantrag werden die Umwelt- (und sozialen) Kosten (wenigstens) auf das Minimum abgegrenzt:

ARTIKEL 1 NUMMER 1 BUCHSTABE B UNTERABSATZ AC A (neu): „externe Kosten": Kosten, die eindeutig vom Straßengütersystem verursacht werden, aber nicht im Marktpreis ihrer Dienstleistungen einkalkuliert sind. Dies können Kosten aufgrund von Verkehrsüberlastung, Umweltkosten, wie lokale und generelle Luftverschmutzung, Lärmbelästigung, Schädigung der Landschaft sowie soziale Kosten, wie Gesundheitskosten und indirekte Kosten im Zusammenhang mit Unfällen, die nicht von Versicherungen abgedeckt werden, sein.

Die folgende Tabelle zeigt die externen Kosten 2000 für Fahrzeuge über 3,5 t hzG auf Autobahnen und Schnellstraßen in Österreich ${ }^{54}$ und ihre monetären Auswirkungen auf die Maut:

\begin{tabular}{|c|c|c|}
\hline \multicolumn{3}{|c|}{$\begin{array}{c}\text { EXTERNE KOSTEN } 2000 \\
\text { für Fahrzeuge über } 3,5 \mathrm{t} \mathrm{hzG} \\
\text { auf Autobahnen und Schnellstraßen } \\
\text { in Österreich }\end{array}$} \\
\hline \multirow{2}{*}{ Kostenart } & Kosten & EUR-Cent \\
\hline & in Mio. EUR & pro Fz-km \\
\hline Unfallkosten & 74 & 2,5 \\
\hline Lärmkosten & 257 & 8,7 \\
\hline Luftverschmutzung - Gesundheitskosten & 246 & 8,3 \\
\hline Luftverschmutzung - Gebäude & 39 & 1,3 \\
\hline Luftverschmutzung - Vegetationschäden & 22 & 0,7 \\
\hline Klimakosten & 174 & 5,9 \\
\hline Summe der externen Kosten & 812 & 27,4 \\
\hline
\end{tabular}

Tabelle 22: Externe Kosten 2000 für Fahrzeuge über 3,5 $\mathrm{t} \mathrm{hzG}$ auf Autobahnen und Schnellstraßen in Österreich

54 HERRY M.: Wegekostenrechnung 2000 für die Autobahnen, Schnellstraßen, Bundesstraßen B, Landes- und Gemeindestraßen in Österreich.

Im Auftrag des Bundesministeriums für Verkehr, Innovation und Technologie, Wien 2001 (Externe Beratung: Dr. Heike Link, DIW Berlin, Prof. Dr. Axhausen, ETH Zürich) 


\subsubsection{Berücksichtigung des Ausweichverkehrs}

Änderungsantrag 3: „Um ein Ausweichen des Verkehrs mit möglicherweise schwerwiegenden Auswirkungen auf die Straßenverkehrssicherheit und die optimale Nutzung des Verkehrsnetzes zu verhindern, müssen die Mitgliedstaaten die Mautgebühren auf allen unmittelbar mit den transeuropäischen Netz konkurrierenden Straßen (Hauptverkehrsstraßennetz) erheben können. In Einklang mit dem Subsidiaritätsprinzip steht es den Mitgliedstaaten sowie - entsprechend ihren jeweiligen Zuständigkeiten - den regionalen und lokalen Behörden frei, unter Beachtung des EG-Vertrags auf anderen, nicht zum Hauptverkehrsstraßennetz gehörenden Straßen, Mautund/oder Benutzungsgebühren zu erheben."

Es wird weiterer Anstrengungen bedürfen, diese eingeleitete Entwicklung fortzusetzen und ehestmöglich einem für die Umweltbelange befriedigenden Ergebnis zuzuführen.

\subsection{Fachtagung, Graz April 2006: Emissionsgesteuerter Güterverkehr}

Nachfolgend sind die Einladung und die Vortragsunterlagen der Fachtagung abgedruckt. 